\title{
SafeChoice: A Novel Approach to Hypergraph Clustering for Wirelength-Driven Placement
}

\author{
Jackey Z. Yan, Chris Chu and Wai-Kei Mak
}

\begin{abstract}
This paper presents a completely new approach to the problem of hypergraph clustering for wirelength-driven placement. The novel algorithm we propose is called SafeChoice (SC). Different from all previous approaches, SC is proposed based on a fundamental theorem, safe condition which guarantees that clustering would not degrade the placement wirelength. To mathematically derive such a theorem, we first introduce the concept of safe clustering, i.e., do clustering without degrading the placement quality. To efficiently check the safe condition for pairwise clustering, we propose a technique called selective enumeration. SafeChoice maintains a global priority queue based on the safeness and area of potential clusters. Using a simple heuristic, it automatically stops clustering when generating more clusters would degrade the placement wirelength. Moreover, we extend SafeChoice to do clustering while considering the object physical locations, i.e, physical clustering. Finally, we apply SafeChoice into a two-phase placement framework and propose a high-quality analytical placement algorithm called SCPlace. Comprehensive experimental results show that the clusters produced by SC consistently help the placer to achieve the best wirelength among all other clustering algorithms, and SCPlace generates the best Half-Perimeter Wirelength compared with all other state-of-theart placers.
\end{abstract}

Index Terms-Hypergraph Clustering, VLSI Placement, Physical Design

\section{INTRODUCTION}

For modern VLSI designs, placement is the most critical stage in the physical synthesis flow. It has significant impacts on timing, routing and even manufacturing. In the nanometer scale era, a circuit typically contains millions of objects. It is extremely challenging for a modern placer to be reasonably fast, yet still be able to produce good solutions. Clustering cuts down the problem size via combining highly connected objects, so that the placers can perform more efficiently and effectively on a smaller problem. It is an attractive solution to cope with the ever-increasing design complexity. Therefore, as an essential approach to improve both the runtime and quality of result, various clustering algorithms have been adopted in the state-of-the-art placement algorithms [1]-[8].

Jackey Z. Yan is with Cadence Design Systems, San Jose, CA 95134 USA (email: zyan@cadence.com).

Chris Chu is with the Department of Electrical and Computer Engineering, Iowa State University, Ames, IA 50010 USA (email: cnchu@iastate.edu).

Wai-Kei Mak is with the Department of Computer Science, National Tsing Hua University, Hsingchu 300, Taiwan (email: wkmak@cs.nthu.edu.tw).

This work was partially supported by IBM Faculty Award, NSF under grant CCF-0540998 and NSC under grant NSC 99-2220-E-007-007.

Copyright (c) 2011 IEEE. Personal use of this material is permitted. However, permission to use this material for any other purposes must be obtained from the IEEE by sending an email to pubs-permissions@ieee.org.

\section{A. Previous Work}

Clustering is a traditional problem in VLSI CAD area. The clustering algorithms proposed long time ago were described in [9]. In the last several years, various new algorithms were proposed to continue improving the clustering quality. In [10] Karypis et al. proposed edge coarsening (EC) clustering. In EC objects are randomly visited. Each object is clustered with the most highly-connected unvisited neighbor object. The connectivity between two objects is computed as the total weight of all edges connecting them with hyperedges represented by a clique model. FirstChoice (FC) clustering was developed in [11] and is very similar to EC. The only difference between them is that for each object in FC, all of its neighbor objects are considered for clustering. FC has been used in placers NTUplace3 [1] and Capo [2]. However, neither EC nor FC considers the impact of cluster size on the clustering quality. Alpert et al. [12] and Chan et al. [13] improved EC and FC respectively, by considering the area of clusters, i.e., clusters with smaller area are preferred to be generated. Cong et al. [14] proposed an edge separabilitybased clustering (ESC). Unlike previous methods, ESC uses edge separability to guide the clustering process. To explore global connectivity information, all edges are ranked via a priority queue (PQ) based on the edge separability. Without violating the cluster size limit, the two objects in the highest ranking edge are clustered. $\mathrm{Hu}$ et al. [15] developed fine granularity (FG) clustering. The difference between FG and ESC is that for FG the order in the PQ is based on edge contraction measured by a mutual contraction metric. FG has been used in placer mFAR [3]. Nam et al. [16] proposed BestChoice (BC) clustering which has been widely used in the top-of-the-line placers APlace [4], mPL6 [5], FastPlace3 [6], RQL [7] and FLOP [8]. Instead of ranking the edges, BC maintains a PQ based on a pair of objects, i.e., each object and its best neighbor object. A score function considering both hyperedge weight and object area is derived to calculate the score between two objects. For each object, the best neighbor object is the neighbor object with the highest score. The two objects at the top of the PQ are clustered iteratively. But updating such a PQ is quite time-consuming. Hence, the authors proposed a lazy-update technique to make a trade-off between the clustering runtime and quality.

All of the above clustering algorithms either explicitly or implicitly transform a hyperedge into a clique model, so that they can handle pair-wise clustering, i.e., cluster two objects at each time. Recently, Li et al. [17] presented NetCluster (NC) that can handle hyperedges directly and cluster more than two objects at one time. In NC, initial clusters are first generated 


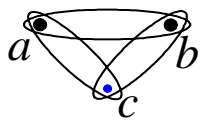

Fig. 1. An example of indirect connections between objects $a$ and $b$.

by FM algorithm [18]. Then a score is assigned to each net. The objects in the net with the highest score are clustered.

For all previous clustering algorithms, none of them specifically aims at improving the placement quality. They proposed a variety of heuristics, e.g., different score functions, to measure the direct connectivity among the objects, so that the most highly directly connected objects are clustered. Of course, the benefit is a reduction of problem size. But, clustering such objects may not help the placer to produce better solution. This is because clustering forces some objects to stay together during placement, which constrains the solution space exploration of the placer. If such constraint is enforced improperly, i.e., clustering objects that should not be clustered, the placement solution would be jeopardized. It has not been proved that clustering highly directly connected objects can definitely minimize the placement wirelength. Even though it makes some sense intuitively to cluster such objects, we believe it is not sufficient to just consider the direct connections. We also need to take the indirect connections into account. For example in Fig. 1, two objects $a$ and $b$ are connected by a two-pin net. At the same time, they are indirectly connected by two two-pin nets via object $c$. Such indirect connections intend to pull $a$ and $b$ towards each other. But they have been ignored in all previous work. As a result, it is very likely that previous algorithms mislead the placers to a low-quality solution.

In order to form the best clusters for placement, we need to solve the fundamental problem of clustering for placement: How to do clustering, so that it can be guaranteed that clustering would not degrade the placement quality?

\section{B. Our Contributions}

This work presents a completely new approach to the problem of hypergraph clustering for wirelength-driven placement. We propose a novel clustering algorithm called SafeChoice $(\mathrm{SC})^{1}$. SC handles hyperedges directly. Different from all previous clustering algorithms, SC is proposed based on a fundamental theorem, which guarantees that clustering would not degrade the placement quality. None of previous techniques has such guarantee. Additionally, three operation modes of SC are presented to achieve various clustering objectives. Essentially, we have seven main contributions:

- Concept of Safe Clustering: We introduce the concept of safe clustering. If clustering some objects would not degrade the wirelength in an optimal placement, it is safe to cluster such objects.

- Safe Condition: Based on the concept of safe clustering, we derive the fundamental theorem - safe condition for

${ }^{1}$ A preliminary version of SafeChoice was presented in [19]. pair-wise clustering. We prove that if any two objects satisfy the safe condition, clustering them would not degrade the wirelength.

- Selective Enumeration: To check the safe condition for pair-wise clustering, we propose selective enumeration. With such method, we can efficiently find out the safe clusters in a circuit.

- SafeChoice: We present SafeChoice algorithm that globally ranks potential clusters via a PQ based on their safeness and area. Iteratively the cluster at the top of the PQ will be formed.

- Smart Stopping Criterion: A smart stopping criterion is proposed based on a simple heuristic. So it can automatically stop clustering once generating more clusters would start to degrade the placement wirelength. As far as we know, none of previous algorithms has such feature.

- Physical SafeChoice: We extend SafeChoice to do clustering if the physical locations of some objects are given. In this way, SafeChoice can make use of such location information, e.g., an initial placement or fixed I/O object locations, and thus produces even better clusters.

- SCPlace: To demonstrate the effectiveness of Physical SafeChoice, we propose a simple and high-quality twophase placement algorithm called SCPlace. SCPlace is simple in the sense that it has only one clustering level and two placement phases. But, it produces significantly better results than all other state-of-the-art placement algorithms.

We compare SC with three state-of-the-art clustering algorithms FC, BC and NC. The results show that the clusters produced by SC consistently helps the placer to generate the best wirelength. Compared with the state-of-the-art placement algorithms, SCPlace is able to generate the best Half-Perimeter Wirelength (HPWL).

The rest of this paper is organized as follows. Section II describes the safe clustering. Section III introduces the algorithm of SafeChoice. Section IV presents the Physical SafeChoice. Section V introduces the algorithm of SCPlace. Experimental results are presented in Section VI. Finally, this paper ends with a conclusion and the direction of future work.

\section{SAfe Clustering}

In this section, we first introduce the concept of safe clustering. Then based on this concept we derive the safe condition for pair-wise clustering. Finally we propose selective enumeration to practically check the safe condition for any two objects in the circuit.

First of all, we introduce some notations used in the discussion. The original netlist is modeled by a hypergraph $G(V, E)$, where $V$ is the set of vertices and $E$ is the set of hyperedges. Given $v \in V, E_{v}$ is the set of hyperedges incident to $v$, and $\bar{E}_{v}=E-E_{v}$. Let $P$ be the set of all possible legalized placements of the vertices in $V$. The wirelength is measured by weighted HPWL.

\section{A. Concept of Safe Clustering}

The concept of safe clustering is defined as follows. 
Definition 1: Safe Clustering: For a set of vertices $V_{c} \subseteq V$ $\left(\left|V_{c}\right| \geq 2\right)$, if the optimal wirelength of the netlist generated by clustering $V_{c}$ is the same as the optimal wirelength of the original netlist, then it is safe to cluster the vertices in $V_{c}$.

The placement problem is NP-hard. In practice we cannot find the optimal wirelength for a real circuit. So we present a more practical definition below.

Definition 2: Safe Clustering ${ }^{\star}: \forall p \in P$, if a set of vertices $V_{c} \subseteq V\left(\left|V_{c}\right| \geq 2\right)$ can be moved to the same location without increasing the wirelength, then it is safe to cluster the vertices in $V_{c}$.

Definition 2 is established based an assumption that the area of every vertex in $V_{c}$ is zero, so that we can move them in a legalized placement and ignore the overlap issue. In other words, we only consider clustering zero-area objects. Definition 2 shows that when safe clustering is performed on any legalized (zero-overlap) placement $p$, it does not increase the total wirelength of $p$. But, the clustering algorithm is not a placement algorithm and thus does not specify how any overlap incurred by the clustering is to be removed. Therefore, we assume that the clusters are small enough, i.e., zero area, compared to the total area of place-able objects in the clustered netlist, such that any wirelength increase incurred by any displacement needed to remove overlap incurred by the clustering does not exceed the wirelength decrease induced by the clustering. This assumption is reasonable, even though there is no zero-area object in the real circuits. This is because for a typical clustering ratio ${ }^{2}$, the size of each cluster is always much smaller than the total area of objects in the circuit. Note that, however, such assumption may not be applicable when some complex floorplan geometry is presented, e.g., when a big cluster is placed in a narrow channel between two big fixed objects. In this case, the displacement needed to remove overlap incurred by the clustering may be quite big.

As you can see, Definition 2 is stronger than Definition 1. If $V_{c}$ is safe for clustering based on Definition 2, it is also safe under Definition 1. In the rest of this paper, we employ Definition 2 for discussion. Based on Definition 2, we derive the definitions for horizontally and vertically safe clustering as follows.

Definition 3: Horizontally/Vertically Safe Clustering: $\forall p \in P$, if a set of vertices $V_{c} \subseteq V\left(\left|V_{c}\right| \geq 2\right)$ can be horizontally/vertically moved to the same $x / y$ coordinate without increasing the wirelength in $x / y$ direction, then it is horizontally/vertically safe to cluster the vertices in $V_{c}$.

Now we show that if vertices in $V_{c}$ are both horizontally and vertically safe for clustering, then it is safe to cluster them under Definition 2. Given any initial placement $p \in P$, firstly we move those vertices horizontally to the same $x$ coordinate. Secondly, we move them vertically to the same $y$ coordinate. Consequently, the vertices in $V_{c}$ are moved to the same location. Based on Definition 3 the wirelength would not increase during the movements. So it is safe to cluster the vertices in $V_{c}$ by Definition 2.

\footnotetext{
${ }^{2}$ The clustering ratio is defined as the ratio of the number of objects in the clustered circuit to the number of objects in the original circuit.
}

In the remaining part of Section II, we consider only $x$ direction and horizontally safe clustering. Analogically, the theoretical proof and mathematical derivation for $y$ direction and vertical safe clustering can be done in a similar way.

\section{B. Safe Condition for Pair-Wise Clustering}

From Definition 2 we derive a condition to mathematically determine whether it is safe to cluster the vertices in $V_{c}$. Firstly, we define two key functions for the derivation. For the sake of simplicity, we always assume $V_{c}$ contains only two vertices $a$ and $b$ (i.e., $V_{c}=\{a, b\}$ ), and $a$ is on the left of $b$.

Definition 4: Wirelength Gradient Function: Given a placement $p \in P$ and a hyperedge $e \in E$, we define

$$
\begin{aligned}
\Delta_{a}(p, e): & \text { Gradient function of wirelength of } e \\
& \text { if } a \text { is moving towards } b . \\
\Delta_{b}(p, e): \text { Gradient function of wirelength of } e & \\
& \text { if } b \text { is moving towards } a .
\end{aligned}
$$

Let $w_{e}\left(w_{e} \geq 0\right)$ be the weight of $e$. From Definition 4 we have

$$
\begin{aligned}
& \Delta_{a}(p, e)= \begin{cases}w_{e} & \text { if } a \text { is the rightmost vertex of } e \\
-w_{e} & \text { if } a \text { is the only leftmost vertex of } e \\
0 & \text { otherwise }\end{cases} \\
& \Delta_{b}(p, e)= \begin{cases}w_{e} & \text { if } b \text { is the leftmost vertex of } e \\
-w_{e} & \text { if } b \text { is the only rightmost vertex of } e \\
0 & \text { otherwise }\end{cases}
\end{aligned}
$$

Considering $a$ is moving towards $b$ in $p$, if $\Delta_{a}(p, e)>0$, it means the wirelength of $e$ will increase; if $\Delta_{a}(p, e)<0$, then the wirelength of $e$ will decrease; otherwise the wirelength of $e$ will not change.

Definition 5: Total wirelength Gradient Function: Given a placement $p \in P$ and $V_{c}=\{a, b\}$, we define

$$
\mathcal{F}_{a b}(p)=\min \left(\sum_{e \in E_{a}} \Delta_{a}(p, e), \sum_{e \in E_{b}} \Delta_{b}(p, e)\right)
$$

In $p$ if both $a$ and $b$ move towards each other, $\mathcal{F}_{a b}(p)$ first calculates the total wirelength change of all hyperedges for moving $a$ and $b$, respectively. Then it returns the one with smaller change. For example, if $\mathcal{F}_{a b}(p)=\sum_{e \in E_{a}} \Delta_{a}(p, e) \leq$ 0 , it means moving $a$ towards $b$ would not increase the total wirelength; if $\mathcal{F}_{a b}(p)>0$, then moving either $a$ or $b$ towards each other would increase the total wirelength. Next, we use this function to derive the safe condition for $a$ and $b$.

Theorem 1: Safe Condition for $V_{c}=\{a, b\}$

It is safe to cluster $a$ and $b$ if $\forall p \in P, \mathcal{F}_{a b}(p) \leq 0$

Proof: Given an initial placement $p^{0} \in P$ with total wirelength $l^{0}$. Because $\forall p \in P, \mathcal{F}_{a b}(p) \leq 0$, we have $\mathcal{F}_{a b}\left(p^{0}\right) \leq 0$. Suppose $\mathcal{F}_{a b}\left(p^{0}\right)=\sum_{e \in E_{a}} \Delta_{a}\left(p^{0}, e\right) \leq 0$. This means by moving $a$ a small distance towards $b$, the total wirelength of all hyperedges would not increase. After such movement, we get another placement $p^{1}$ with total wirelength $l^{1}$, where $l^{0} \geq l^{1}$. For $p^{1}$ we still have $\mathcal{F}_{a b}\left(p^{1}\right) \leq 0$. Suppose this time $\mathcal{F}_{a b}\left(p^{1}\right)=\sum_{e \in E_{b}} \Delta_{b}\left(p^{1}, e\right) \leq 0$. This means 
moving $b$ a small distance towards $a$ would not increase the total wirelength. Again, after such movement, we get another placement $p^{2}$ with total wirelength $l^{2}$, where $l^{1} \geq l^{2}$. We keep moving either $a$ or $b$ towards each other until they reach the same location. Suppose the final total wirelength is $l^{n}$. Because after each movement we always have $\mathcal{F}_{a b}(p) \leq 0$, which means the total wirelength would not increase, eventually we have $l^{0} \geq l^{n}$.

As a result, given any initial placement $p^{0}$ we can gradually move $a$ and $b$ to the same location without increasing the wirelength. So based on Definition 2, it is safe to cluster vertices $a$ and $b$.

\section{Selective Enumeration}

To check whether it is safe to cluster $a$ and $b$, Theorem 1 shows that we need to generate all placements in $P$. To do so, we have to enumerate all possible positions for all vertices in $V$. Apparently this is not a practical approach. In this section, we show that in order to check Theorem 1, it is sufficient to consider only a small subset of placements. Selective enumeration technique is proposed to enumerate such necessary placements.

Selective enumeration is motivated by the following principle: Given two placements $p_{1}, p_{2} \in P$, if we know $\mathcal{F}_{a b}\left(p_{1}\right) \leq$ $\mathcal{F}_{a b}\left(p_{2}\right)$, then $p_{1}$ can be ignored in the enumeration. This is because Theorem 1 shows that the safe condition is only determined by the placement with the maximum $\mathcal{F}_{a b}(p)$ value. So the basic idea of selective enumeration is to find out the relationship of $\mathcal{F}_{a b}(p)$ values among different placements, so that in the enumeration process we can ignore the placements with smaller or equal $\mathcal{F}_{a b}(p)$ values. Placements in $P$ are generated by different positions of different vertices. Our goal is to identify some vertices in $V$, such that some or even all of their possible positions can be ignored.

We first classify the vertices in $V$ into two categories $V_{\bar{a} \bar{b}}$ and $V_{a b}\left(V_{\bar{a} \bar{b}} \cup V_{a b} \cup\{a, b\}=V\right)$. Then we discuss the enumeration of their positions separately. $\forall v \in V, x_{v}$ denotes the $x$ coordinate of $v$.

1) $V_{\bar{a} \bar{b}}$ : vertices connecting with neither $a$ nor $b$.

2) $V_{a b}$ : vertices connecting with at least one of $a$ and $b$.

Lemma 1: Given a placement $p \in P$, by moving vertex $v \in V_{\bar{a} \bar{b}}$ to any other position, another placement $p^{\prime} \in P$ is generated. We have $\mathcal{F}_{a b}(p)=\mathcal{F}_{a b}\left(p^{\prime}\right)$.

Proof: Since $\forall v \in V_{\bar{a} \bar{b}}, v$ connects with neither $a$ nor $b$, changing the position of $v$ would not change the leftmost or rightmost vertex of any hyperedge connecting with $a$ or $b$. Therefore,

$$
\begin{aligned}
& \forall e \in E_{a}, \Delta_{a}(p, e)=\Delta_{a}\left(p^{\prime}, e\right) \\
& \forall e \in E_{b}, \Delta_{b}(p, e)=\Delta_{b}\left(p^{\prime}, e\right)
\end{aligned}
$$

Thus, $\mathcal{F}_{a b}(p)=\mathcal{F}_{a b}\left(p^{\prime}\right)$.

Based on Lemma 1, in the enumeration we can simply ignore all vertices in $V_{\bar{a} \bar{b}}$.

Lemma 2: Given a placement $p \in P$, vertex $v \in V_{a b}$ and $x_{v}=k_{1}$. After moving $v$ to $x_{v}=k_{2}$, another placement $p^{\prime} \in P$ is generated. We have $\mathcal{F}_{a b}(p)=\mathcal{F}_{a b}\left(p^{\prime}\right)$ if any one of the following conditions is satisfied: (1) $k_{1} \leq x_{a}$ and $k_{2} \leq x_{a}$; (2) $k_{1} \geq x_{b}$ and $k_{2} \geq x_{b}$; (3) $x_{a}<k_{1}<x_{b}$ and $x_{a}<k_{2}<x_{b}$.

Proof: Suppose condition (1) holds, i.e., $v$ is on the left of $a$ in both $p$ and $p^{\prime}$. $\forall e \in E_{v}$, we consider two ${ }^{3}$ possible values of $\Delta_{a}(p, e)$ :

- $\Delta_{a}(p, e)=w_{e}$

This means $a$ is the rightmost vertex of $e$ in $p$. After moving $v$ to $k_{2}$, because $k_{2} \leq x_{a}, a$ is still the rightmost vertex of $e$ in $p^{\prime}$. Thus, $\Delta_{a}\left(p^{\prime}, e\right)=w_{e}=\Delta_{a}(p, e)$.

- $\Delta_{a}(p, e)=0$

This means $a$ is neither the only leftmost nor the rightmost vertex of $e$ in $p$. After moving $v$ to $k_{2}$, because $k_{2} \leq x_{a}, v$ is still on the left of $a$ in $p^{\prime}$. Thus, $\Delta_{a}\left(p^{\prime}, e\right)=0=\Delta_{a}(p, e)$.

So $\forall e \in E_{v}, \Delta_{a}(p, e)=\Delta_{a}\left(p^{\prime}, e\right)$. Similarly we have $\forall e \in$ $E_{v}, \Delta_{b}(p, e)=\Delta_{b}\left(p^{\prime}, e\right)$. Therefore,

$$
\begin{aligned}
& \forall e \in E_{a}, \Delta_{a}(p, e)=\Delta_{a}\left(p^{\prime}, e\right) \\
& \forall e \in E_{b}, \Delta_{b}(p, e)=\Delta_{b}\left(p^{\prime}, e\right)
\end{aligned}
$$

Thus, $\mathcal{F}_{a b}(p)=\mathcal{F}_{a b}\left(p^{\prime}\right)$. Analogically, the cases for conditions (2) and (3) can be proved as well.

Lemma 2 shows that $\forall v \in V_{a b}$, instead of enumerating all possible positions, we only need to consider three possibilities: (1) $v$ is on the left of $a\left(x_{v} \leq x_{a}\right)$; (2) $v$ is on the right of $b$ $\left(x_{v} \geq x_{b}\right)$; (3) $v$ is between $a$ and $b\left(x_{a}<x_{v}<x_{b}\right)$.

Based on Lemma 1 and 2, we need to enumerate $3^{\left|V_{a b}\right|}$ different placements rather than all placements in $P$. Next, we will further cut down this number from $3^{\left|V_{a b}\right|}$ to $2^{\left|V_{a b}\right|}$, by ignoring all positions between $a$ and $b$.

Lemma 3: Given a placement $p \in P$, such that vertex $v \in$ $V_{a b}$ is between $a$ and $b\left(x_{a}<x_{v}<x_{b}\right)$. After moving $v$ either to the left of $a$ or to the right of $b$, another placement $p^{\prime} \in P$ is generated. We have $\mathcal{F}_{a b}(p) \leq \mathcal{F}_{a b}\left(p^{\prime}\right)$.

Proof: Suppose $v$ is moved to the left of $a$.

For $a$, after the movement, $a$ might become the rightmost vertex of some hyperedge. So we have

$$
\forall e \in E_{v}, \Delta_{a}(p, e) \leq \Delta_{a}\left(p^{\prime}, e\right)
$$

For $b$, after the movement, $v$ is still on the left of $b$. So we have

$$
\forall e \in E_{v}, \Delta_{b}(p, e)=\Delta_{b}\left(p^{\prime}, e\right)
$$

Based on Equations 1-2, we have

$$
\begin{aligned}
& \forall e \in E_{a}, \Delta_{a}(p, e) \leq \Delta_{a}\left(p^{\prime}, e\right) \\
& \forall e \in E_{b}, \Delta_{b}(p, e)=\Delta_{b}\left(p^{\prime}, e\right)
\end{aligned}
$$

Thus, $\mathcal{F}_{a b}(p) \leq \mathcal{F}_{a b}\left(p^{\prime}\right)$. Similarly, we can prove the case for $v$ is moved to the right of $b$.

So far, we have proved that we only need to consider two possible positions (on the left of $a$ and on the right of $b$ ) for each vertex in $V_{a b}$, i.e., totally $2^{\left|V_{a b}\right|}$ different placements. In a modern circuit, $\left|V_{a b}\right|$ may become more than 1000 . So

\footnotetext{
${ }^{3}$ Because $v$ is on the left of $a, a$ would not be the only leftmost vertex of $e$. Thus, $\Delta_{a}(p, e) \neq-w_{e}$.
} 


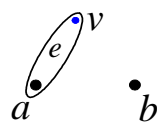

( I )

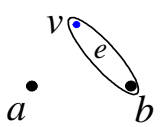

( II )

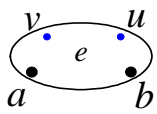

( III )
Fig. 2. Simple examples of vertices that can be fixed.

practically $2^{\left|V_{a b}\right|}$ is still too big to enumerate. Therefore, we intend to further cut down this number.

We notice that for some vertices in $V_{a b}$, it is not always necessary to consider both of the two possible positions. For example in Fig. 2-(I), $v$ is only connected with $a$ via $e$. If $v$ is on the left of $a$ in placement $p_{l}$, then $\mathcal{F}_{a b}\left(p_{l}\right)=\min \left(w_{e}, 0\right)=$ 0 ; if $v$ is on the right of $b$ in placement $p_{r}$, then $\mathcal{F}_{a b}\left(p_{r}\right)=$ $\min \left(-w_{e}, 0\right)=-w_{e}$. We have $\mathcal{F}_{a b}\left(p_{l}\right)>\mathcal{F}_{a b}\left(p_{r}\right)$. So, we can ignore $p_{r}$ where $v$ is on the right of $b$. To make use of such property and further reduce the enumeration size, in the following part we identify three subsets of vertices in $V_{a b}\left(V^{I}\right.$, $V^{I I}$ and $V^{I I I}$ ), and prove that under certain condition the positions of those vertices can be fixed in the enumeration. Let $N_{a}$ denote the set of vertices sharing at least one hyperedge with vertex $a$, and $\bar{N}_{a}=V-N_{a}$. Similarly, we can define $N_{b}$ and $\bar{N}_{b}$.

I. $V^{I}=N_{a} \cap \bar{N}_{b}$ ( e.g., in Fig. 2-(I) vertex $v \in V^{I}$ )

II. $V^{I I}=\bar{N}_{a} \cap N_{b}$ ( e.g., in Fig. 2-(II) vertex $v \in V^{I I}$ )

III. $V^{I I I}=\left\{v \mid v \in V_{a b}\right.$ s.t. $\left.\left(E_{v} \cap\left(E_{a} \cup E_{b}\right)\right) \subset\left(E_{a} \cap E_{b}\right)\right\}$ ( e.g., in Fig. 2-(III) vertices $v, u \in V^{I I I}$ )

Lemma 4: Given a placement $p \in P$, such that vertex $v \in$ $V^{I}$ is on the left of $a$. After moving $v$ to the right of $b$, another placement $p^{\prime} \in P$ is generated. We have $\mathcal{F}_{a b}(p) \geq \mathcal{F}_{a b}\left(p^{\prime}\right)$.

Proof: Let $E_{v \cap a b}=E_{v} \cap\left(E_{a} \cup E_{b}\right)$.

- In placement $p, \forall e \in E_{v \cap a b}$, we consider two cases:

- $\exists$ vertex $c \in e(c \neq a, c \neq v)$, s.t. $x_{c} \geq x_{b}$

Because $x_{v} \leq x_{a}$ and $x_{c} \geq x_{b}, x_{v} \leq x_{a} \leq x_{c} . a$ is neither the only leftmost nor the rightmost vertex of $e$. So $\Delta_{a}(p, e)=0$.

- $\nexists$ vertex $c \in e(c \neq a, c \neq v)$, s.t. $x_{c} \geq x_{b}$

Because $x_{v} \leq x_{a}$ and no other vertices in $e$ are on the right of $b, a$ is the rightmost vertex of $e$. So $\Delta_{a}(p, e)=w_{e}$.

Thus, $\forall e \in E_{v \cap a b}, \Delta_{a}(p, e) \geq 0$.

- In placement $p^{\prime}, \forall e \in E_{v \cap a b}$, we consider two cases:

- $\exists$ vertex $c \in e(c \neq a, c \neq v)$, s.t. $x_{c} \leq x_{a}$

Because $x_{v}^{\prime} \geq x_{b}$ and $x_{c} \leq x_{a}, x_{c} \leq x_{a} \leq x_{v}^{\prime} . a$ is neither the only leftmost nor the rightmost vertex of $e$. So $\Delta_{a}\left(p^{\prime}, e\right)=0$.

- $\nexists$ vertex $c \in e(c \neq a, c \neq v)$, s.t. $x_{c} \leq x_{a}$

Because $x_{v}^{\prime} \geq x_{b}$ and no other vertices in $e$ are on the left of $a, a$ is the only leftmost vertex of $e$. So $\Delta_{a}(p, e)=-w_{e}$.

Thus, $\forall e \in E_{v \cap a b}, \Delta_{a}\left(p^{\prime}, e\right) \leq 0$.

So $\forall e \in E_{v \cap a b}, \Delta_{a}(p, e) \geq \Delta_{a}\left(p^{\prime}, e\right)$. Also $\forall v \in V^{I}$, $v$ does not connect with $b$, so $\forall e \in E_{v \cap a b}, \Delta_{b}(p, e)=\Delta_{b}\left(p^{\prime}, e\right)$.
Therefore,

$$
\begin{aligned}
& \forall e \in E_{a}, \Delta_{a}(p, e) \geq \Delta_{a}\left(p^{\prime}, e\right) \\
& \forall e \in E_{b}, \Delta_{b}(p, e)=\Delta_{b}\left(p^{\prime}, e\right)
\end{aligned}
$$

Thus, $\mathcal{F}_{a b}(p) \geq \mathcal{F}_{a b}\left(p^{\prime}\right)$.

From Lemma 4, $\forall v \in V^{I}$ we can fix $v$ on the left of $a$. As $V^{I I}$ is symmetrical with $V^{I}$, similarly we can prove that $\forall v \in V^{I I}$ we can fix $v$ on the right of $b$.

Lemma 5: Given a placement $p \in P$, such that vertex $v \in$ $V^{I I I}$ is on the left of $a$, vertex $u \in V^{I I I}$ is on the right of $b$, and $E_{v} \cap\left(E_{a} \cup E_{b}\right)=E_{u} \cap\left(E_{a} \cup E_{b}\right)$. After moving either one or both of them to another position, i.e., moving $v$ to the right of $b$ and $u$ to the left of $a$, another placement $p^{\prime}$ is generated. We have $\mathcal{F}_{a b}(p) \geq \mathcal{F}_{a b}\left(p^{\prime}\right)$.

Proof: Let $E_{v-u}=E_{v} \cap\left(E_{a} \cup E_{b}\right)=E_{u} \cap\left(E_{a} \cup E_{b}\right)$. We consider all three possible movements of $v$ and $u$.

- $v$ moved to the right of $b, u$ did not move

In placement $p^{\prime}, \forall e \in E_{v-u}$ we consider two cases:

- $\exists$ vertex $c \in e(c \neq a)$, s.t. $x_{c} \leq x_{a}$

In this case, $a$ is neither the only leftmost nor the rightmost vertex in $e$, and $b$ is neither the leftmost nor the only rightmost vertex in $e$. So $\Delta_{a}\left(p^{\prime}, e\right)=0$, $\Delta_{b}\left(p^{\prime}, e\right)=0$.

- $\nexists$ vertex $c \in e(c \neq a)$, s.t. $x_{c} \leq x_{a}$ In this case, $a$ is the only leftmost vertex in $e$, and $b$ is neither the leftmost nor the only rightmost vertex in $e$. So $\Delta_{a}\left(p^{\prime}, e\right)=-w_{e}, \Delta_{b}\left(p^{\prime}, e\right)=0$.

- $u$ moved to the left of $a, v$ did not move

In placement $p^{\prime}, \forall e \in E_{v-u}$ we consider two cases:

- $\exists$ vertex $c \in e(c \neq b)$, s.t. $x_{c} \geq x_{b}$

In this case, $a$ is neither the only leftmost nor the rightmost vertex in $e$, and $b$ is neither the leftmost nor the only rightmost vertex in $e$. So $\Delta_{a}\left(p^{\prime}, e\right)=0$, $\Delta_{b}\left(p^{\prime}, e\right)=0$.

- $\nexists$ vertex $c \in e(c \neq b)$, s.t. $x_{c} \geq x_{b}$

In this case, $b$ is the only rightmost vertex in $e$, and $a$ is neither the only leftmost nor the rightmost vertex in $e$. So $\Delta_{a}\left(p^{\prime}, e\right)=0, \Delta_{b}\left(p^{\prime}, e\right)=-w_{e}$.

- $v$ moved to the right of $b, u$ moved to the left of $a$ In this case, $a$ is neither the only leftmost nor the rightmost vertex in $e$, and $b$ is neither the leftmost nor the only rightmost vertex in $e$. So $\forall e \in E_{v-u}, \Delta_{a}\left(p^{\prime}, e\right)=0$, $\Delta_{b}\left(p^{\prime}, e\right)=0$.

For all of the above cases, $\Delta_{a}\left(p^{\prime}, e\right) \leq 0$ and $\Delta_{b}\left(p^{\prime}, e\right) \leq 0$. In placement $p, \forall e \in E_{v-u}$ because $a$ is neither the only leftmost nor the rightmost vertex in $e$, and $b$ is neither the leftmost nor the only rightmost vertex in $e$, we have $\Delta_{a}(p, e)=\Delta_{b}(p, e)=$ 0 . As a result, we have $\forall e \in E_{v-u}, \Delta_{a}(p, e) \geq \Delta_{a}\left(p^{\prime}, e\right)$, $\Delta_{b}(p, e) \geq \Delta_{b}\left(p^{\prime}, e\right)$, Therefore,

$$
\begin{aligned}
& \forall e \in E_{a}, \Delta_{a}(p, e) \geq \Delta_{a}\left(p^{\prime}, e\right) \\
& \forall e \in E_{b}, \Delta_{b}(p, e) \geq \Delta_{b}\left(p^{\prime}, e\right)
\end{aligned}
$$

Thus, $\mathcal{F}_{a b}(p) \geq \mathcal{F}_{a b}\left(p^{\prime}\right)$.

Lemma 5 shows that if $\exists v, u \in V^{I I I}$ and $E_{v} \cap\left(E_{a} \cup E_{b}\right)=$ $E_{u} \cap\left(E_{a} \cup E_{b}\right)$, then we can fix $v$ to the left of $a$ and $u$ to the right of $b$. 


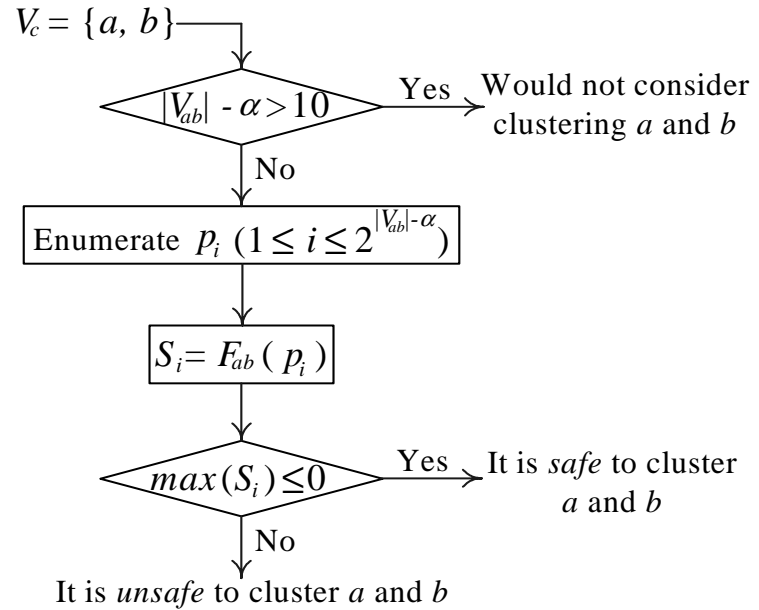

Fig. 3. The flow of selective enumeration.

In all, we have identified three subsets of vertices in $V_{a b}$. If certain condition is satisfied, those vertices can be fixed in the enumeration. Note that those three subsets may not include all vertices that can be fixed in $V_{a b}$. We believe more complicated subsets and conditions can be derived. But for the sake of simplicity, SafeChoice considers only the above three subsets.

Let the total number of vertices in $V^{I}, V^{I I}$ and $V^{I I I}$ be $\alpha$. As a result, given two objects $a$ and $b$, we only need to enumerate $L=2^{\left|V_{a b}\right|-\alpha}$ different placements. For each of those enumerated placement $p_{i}(1 \leq i \leq L)$, we calculate a score $s_{i}=\mathcal{F}_{a b}\left(p_{i}\right)$. We define

$$
s_{\max }=\max \left(s_{1}, s_{2}, \ldots, s_{L}\right)
$$

Based on Theorem 1, if $s_{\max } \leq 0$, then it is safe to cluster $a$ and $b$. The flow of selective enumeration is shown in Fig. 3.

The more placements we enumerate (i.e., the bigger $L$ is), the slower the algorithm runs. To limit the runtime, at most $2^{10}$ placements are enumerated by default. If $\left|V_{a b}\right|-\alpha>$ 10 , we simply would not consider clustering $a$ and $b$, and consequently we may lose some potential safe clusters. Table I shows the number of cases where $\left|V_{a b}\right|-\alpha<=10$ and $\left|V_{a b}\right|-$ $\alpha>10$, and also the maximum value of $\left|V_{a b}\right|-\alpha$ for each ISPD 05/06 circuit. As you can see, in practice we have $\left|V_{a b}\right|-$ $\alpha \leq 10$ for most of the pairs (i.e., more than $90 \%$ of the pairs). Even if the unconsidered pairs are all safe, we would only lose a very small portion of safe clusters.

\section{Algorithm of SAFEChoice}

In the previous section, we have described a practical method of checking the safe condition for pair-wise clustering. As shown in Definition 3, the safe condition has to be checked both horizontally and vertically. However, without considering fixed vertices, e.g., I/O objects, if vertices in $V_{c}$ are horizontally safe for clustering, then they are always vertically safe for clustering as well. This is because a vertical movement in a placement $p$ is the same as a horizontal movement in another placement obtained by rotating $p$ by $90^{\circ}$. If a set of vertices is horizontally safe for clustering, it is also vertically
TABLE I

PROFILE OF SELECTIVE ENUMERATION FOR EACH CIRCUIT.

\begin{tabular}{|c|c|c|c|}
\hline Circuit & $\begin{array}{c}\text { Number of Cases } \\
\end{array}$ & $\begin{array}{c}\text { Number of Cases } \\
\left|V_{a b}\right|-\alpha<=10(\%)\end{array}$ & $\begin{array}{c}\text { Maximum } \\
\left|V_{a b}\right|-\alpha>10(\%)\end{array}$ \\
\hline \hline adaptec1 & $3079149(94 \%)$ & $205937(6 \%)$ & 78 \\
\hline adaptec2 & $2834333(92 \%)$ & $252967(8 \%)$ & 110 \\
\hline adaptec3 & $5568171(90 \%)$ & $591043(10 \%)$ & 191 \\
\hline adaptec4 & $6362256(93 \%)$ & $448166(7 \%)$ & 110 \\
\hline bigblue1 & $3677691(94 \%)$ & $218500(6 \%)$ & 154 \\
\hline bigblue2 & $6941283(95 \%)$ & $351816(5 \%)$ & 1582 \\
\hline bigblue3 & $12136725(95 \%)$ & $592998(5 \%)$ & 205 \\
\hline bigblue4 & $22954456(92 \%)$ & $2104452(8 \%)$ & 1102 \\
\hline adaptec5 & $9935184(90 \%)$ & $1058753(10 \%)$ & 278 \\
\hline newblue1 & $4610210(97 \%)$ & $159220(3 \%)$ & 608 \\
\hline newblue2 & $4058091(98 \%)$ & $73838(2 \%)$ & 144 \\
\hline newblue3 & $6758853(93 \%)$ & $489111(7 \%)$ & 1016 \\
\hline newblue4 & $9215574(95 \%)$ & $530334(5 \%)$ & 331 \\
\hline newblue5 & $12046236(94 \%)$ & $744213(6 \%)$ & 372 \\
\hline newblue6 & $15996670(91 \%)$ & $1527619(9 \%)$ & 1316 \\
\hline newblue7 & $26414433(94 \%)$ & $1700947(6 \%)$ & 1151 \\
\hline
\end{tabular}

safe for clustering, which means it is sufficient to check the safe condition only in $x$ direction. Therefore, in this section we ignore the fixed objects by treating them the same as movable ones, apply selective enumeration in a PQ-based algorithm flow and propose SafeChoice algorithm. To satisfy various clustering objectives, we present three operation modes for SafeChoice.

\section{A. Priority-Queue Based Framework}

Previous work [12], [13] show that the cluster size has significant impacts on the clustering quality. If two potential clusters have the same connectivity information, the one with the smaller area is preferred to be formed first. Moreover, because the concept of safe clustering in Definition 2 is defined based on the assumption of clustering zero-area objects, to apply such concept on real circuits we need relax this assumption and cluster small objects ${ }^{4}$. Thus, in SafeChoice to balance the safeness and area, we use the following cost function to calculate the cost $C$ for clustering two objects $a$ and $b$.

$$
C(a, b)=S^{*}+\theta \times \frac{A_{a}+A_{b}}{\bar{A}_{s}}
$$

where $\theta$ is the weight between the safeness and area (based on the experiments $\theta=4$ by default), $A_{a}$ and $A_{b}$ denote the area of $a$ and $b$ respectively, $\bar{A}_{s}$ is the average standard cell area in a circuit, and $S^{*}$ is a term describing the safeness of clustering $a$ and $b . S^{*}$ is calculated based on different modes of SafeChoice (see Section III-B).

In SafeChoice we maintain a global PQ similar to that in [16]. But we rank each pair of objects based on the cost obtained by Equation 4. If two pairs have the same cost, we just randomly determine the priority between them. For SafeChoice, it is time-consuming to consider all possible pairs in $V$. So for each object, we only consider its neighbor objects connected by the nets containing at most $\beta$ objects (based on the experiments $\beta=7$ by default). Iteratively, SafeChoice clusters the pair of objects at the top of the PQ, and then update the PQ using lazy-update. For different operation

\footnotetext{
${ }^{4}$ After relaxing the assumption, moving the small objects may create small overlap. But as the objects are small, the result placement can be legalized by slightly shifting objects around and the impact to HPWL should be minimal
} 
TABLE II

DIFFERENCES OF THREE MODES (SC IS THE DEFAULT MODE).

\begin{tabular}{|c|c|c|c|}
\hline Mode & Clustering Objective & $S^{*}$ & Stopping Criterion \\
\hline \hline SC-G & safe clusters guarantee & $s_{\max }$ & no more safe clusters is in PQ \\
\hline SC-R & target clustering ratio & $\bar{s}$ & target clustering ratio is reached \\
\hline SC & best placement wirelength & $\bar{s}$ & threshold cost $C_{t}$ is reached \\
\hline
\end{tabular}

modes, SafeChoice stops clustering based on different stopping criteria, which will be addressed in Section III-B.

\section{B. Operation Modes of SafeChoice}

Given a circuit, some algorithms (e.g., FC and BC) can reach any clustering ratio $\gamma$, while others (e.g., FG and NC) can only reach a certain $\gamma$. None of previous work is able to automatically stop clustering when the best $\gamma$ is reached. By default SafeChoice automatically stops clustering when generating more clusters would degrade the placement wirelength. Additionally, to achieve other clustering objectives, e.g., any target $\gamma$, SafeChoice is capable of performing under various modes (see Table II):

\section{- Safety Guarantee Mode [SC-G]}

SC-G aims at producing the completely safe clusters. Under this mode, $S^{*}=s_{\max }$ in Equation 4. In each iteration, we cluster the pair of objects at the top of the PQ only if its $S^{*} \leq 0$. Based on Theorem 1, we guarantee that the formed clusters are safe. SC-G terminates when there is no such safe clusters in the PQ.

\section{- Clustering Ratio Mode [SC-R]}

The SC-G mode may not achieve low clustering ratio in practice, because the number of safe clusters in a circuit is usually limited. Sometimes if clustering cannot significantly reduce the circuit size, even though all clusters are safe, the placer may not perform efficiently and produce better result. So to make a trade-off between safeness and circuit size reduction, SC-R produces some unsafe clusters, besides the safe ones. We derive the following function to evaluate the safeness of each cluster:

$$
\bar{s}=\frac{\sum_{i=1}^{L} s_{i}}{L}
$$

Basically, for a pair of objects $a$ and $b$ Equation 5 calculates the average score $\bar{s}$ over the $L$ enumerated placements. Under SC-R mode, $S^{*}=\bar{s}$ in Equation 4. Iteratively, SC-R clusters the pair of objects at the top of the PQ until the target $\gamma$ is reached.

\section{- Smart Mode [SC] (default mode)}

Using a simple heuristic, the smart mode stops the clustering process when a typical placer achieves the best placement wirelength. None of previous clustering algorithms has such feature. For different circuits, the $\gamma$ for the best placement wirelength may be different. In $\mathrm{SC}$, we set a threshold cost $C_{t}$, and use the same cost function as in SC-R. During the clustering process, SC would not terminate until the cost reaches $C_{t}$. Based on the experimental results, we set $C_{t}=21$ by default. With this simple heuristic, SC is able to automatically stop when generating more clusters starts to degrade the placement wirelength.

\section{Physical SAFeChoice}

In this section, we extend SafeChoice to do clustering while considering the object physical locations, i.e., physical clustering.

Compared with non-physical clustering algorithms, physical clustering is to do clustering based on both the netlist connectivity information and the object physical locations. Such physical locations can be obtained from an initial placement or existing fixed objects. It has been shown in [3], [6], [7], [20] that the physical clustering can significantly improve the clustering quality. For SafeChoice, it is very natural to be extended to physical clustering. This is because SafeChoice applies selective enumeration to enumerate different placements. If an initial placement is given, many more placements can be ignored in the enumeration. This simplifies the enumeration process by pruning away the placements that would not be possibly generated.

In the following subsections, we first introduce the safe condition for Physical SafeChoice. After that, we present how to further reduce the enumeration size based on the given physical information. Finally, we show the corresponding changes of cost functions in different modes of Physical SafeChoice.

\section{A. Safe Condition for Physical SafeChoice}

Because in Physical SafeChoice the fixed objects are taken into account, horizontally safe may not always imply vertically safe. Therefore, we need to consider the safe condition for both $x$ and $y$ directions in Physical SafeChoice.

Definitions 4 and 5 are defined for $x$ direction. But they can be easily extended to $y$ direction. Based on Theorem 1, we present the safe condition for Physical SafeChoice as follows.

Theorem 2: Safe Condition for $V_{c}=\{a, b\}$ in Physical SafeChoice

It is safe to cluster $a$ and $b$ if

$$
\forall p \in P, \mathcal{F}_{a b}^{x}(p) \leq 0 \text { and } \mathcal{F}_{a b}^{y}(p) \leq 0
$$

where $\mathcal{F}_{a b}^{x}(p)$ and $\mathcal{F}_{a b}^{y}(p)$ are the total wirelength gradient functions for $x$ and $y$ directions, respectively. In Section II-A we have proved that if vertices in $V_{c}$ are both horizontally and vertically safe for clustering, then it is safe to cluster them. Therefore, Theorem 2 can be proved similarly as in Theorem 1 .

\section{B. Enumeration Size Reduction based on Physical Location}

In this subsection, we use the physical location information to cut down the placement enumeration size. In this following discussion, we assume that such physical locations are derived from an initial placement.

First of all, for each object we define a square-shape region to differentiate between the "long" and "short" distances (in one dimension) of two objects. The center of each squareshape region is the corresponding object location in the initial placement. The insight is that we assume in the final placement the objects would not be placed outside of their regions. So intuitively, the better the initial placement is, the less 


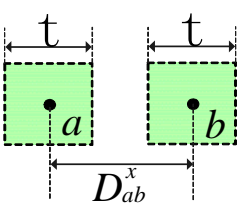

(a)

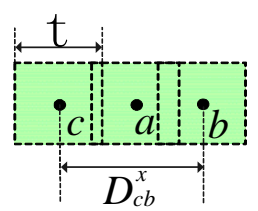

(b)

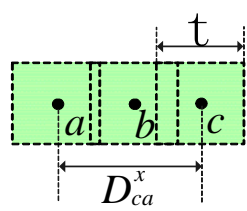

(c)
Fig. 4. Examples of three scenarios with square-shape region.

displacements of object locations between the initial and final placements we have, and thus the smaller such regions are. Let $t$ denote the side length of each square-shape region. $\forall v, u \in V, D_{v u}^{x}$ denotes the distance of two vertices $v$ and $u$ in $x$ direction. Therefore, based on the square-shape region, we can derive the following three scenarios in $x$ direction to cut down the enumeration size (the scenarios for $y$ direction can be derived similarly).

1) If $D_{a b}^{x}>t$, then we would not consider to cluster $a$ and b. (see Fig. 4-(a))

2) If $x_{c} \leq x_{a}$ and $D_{c b}^{x}>t$, then in selective enumeration we fix $c$ on the left of $a$ (see Fig. 4-(b));

3) If $x_{c} \geq x_{b}$ and $D_{c a}^{x}>t$, then in selective enumeration we fix $c$ on the right of $b$ (see Fig. 4-(c));

Scenario 1) is used as a filter to prune away the pairs of objects that would not be clustered due to the "long" distance between them. Scenarios 2) and 3) are applied within selective enumeration to identify the subsets of vertices that can be fixed. In Scenario 2) the reason why we consider " $D_{c b}^{x}>t$ ", instead of " $D_{c a}^{x}>t$ ", is that as long as $c$ is unlikely to be on the right of $b$, it will definitely be on the left of $a$. This is because as shown in Lemma 3, there only two possible locations to enumerate for $c$, i.e., on the left of $a$ and on the right of $b$. The same reason applies for " $D_{c a}^{x}>t$ " in Scenario $3)$.

Ideally, for different objects $t$ should be different. However, finding such a $t$ accurately for each object is still an open problem. After all, we just need a simple estimation on the displacement that is roughly determined by the quality of the placer. Therefore, by default we set $t=15 \times h_{\text {row }}$ based on the experiments and our experience on placement, where $h_{\text {row }}$ is the placement row height.

\section{Cost Function for Physical SafeChoice}

In Physical SafeChoice, we employ almost the same cost function as Equation 4. However, the $S^{*}$ term in Equation 4 is defined based on one dimension, i.e., $x$ direction. So we need to extend it to two dimensions. The $S^{*}$ for the three operation modes in Physical SafeChoice are listed in Table III, where $s_{\max }^{x}$ and $\bar{s}^{x}$ denote the $s_{\max }$ (Equation 3) and $\bar{s}$ (Equation 5) for $x$ direction respectively, similarly for $s_{\max }^{y}$ and $\bar{s}^{y}$.

\section{SafeChoice-Based Two-Phase Placement}

In this section, we first propose a simple two-phase placement flow. Then based on this new algorithm flow, we present SCPlace, a high-quality analytical placement algorithm.
TABLE III

$S^{*}$ FOR THE THREE MODES IN PHySICAL SAFEChoice.

\begin{tabular}{|c|c|}
\hline Mode & $S^{*}$ \\
\hline \hline SC-G & $\max \left(s_{\max }^{x}, s_{\max }^{y}\right)$ \\
\hline SC-R & $\left(\bar{s}^{x}+\bar{s}^{y}\right) / 2$ \\
\hline SC & $\left(\bar{s}^{x}+\bar{s}^{y}\right) / 2$ \\
\hline
\end{tabular}

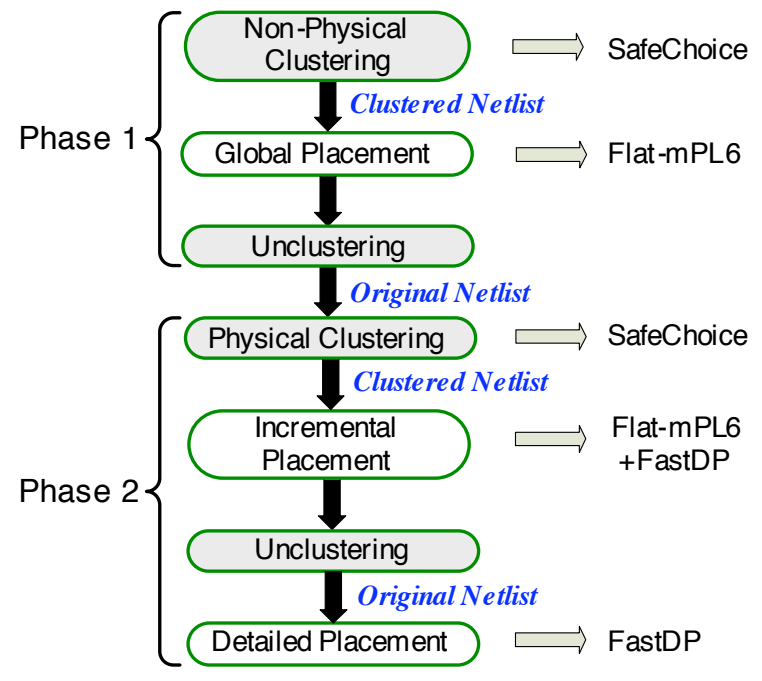

Fig. 5. The simple two-phase placement flow in SCPlace.

The state-of-the-art placement algorithms [1]-[7] all adopt a multilevel framework to cope with the ever-increasing complexities of modern VLSI placement. At each hierarchical level of the coarsening phase, the placers first do clustering on the netlist passed from previous level, and then do placement of the clustered netlist. Such a multilevel clustering-placement process does not stop, until the original circuit is reduced to a reasonably smaller size. Subsequently, a corresponding multilevel unclustering-placement process is applied at the uncoarsening phase. Typically, modern placers contain at least four levels of clustering and placement.

Different from previous work, we propose a simple twophase placement flow shown in Fig. 5. It is simple in the sense that this flow contains only one level of clustering and two phases of placement. The goal of the first phase is to generate an initial placement, and to provide the physical location information for the physical clustering in the next phase. Then at the second phase, we apply physical clustering on the original netlist rather than the clustered netlist from previous clustering. This is the key difference between our physical clustering scheme and the ones in [3], [6], [7], [20]. The reason for doing this is that non-physical clustering may produce some low-quality clusters due to the lack of physical information. In order to correct such mistake in non-physical clustering, in physical clustering we form the clusters from the original netlist. The location of each such cluster is calculated as the average location of all objects in that cluster. As a result, after physical clustering we have an updated location for each object in the clustered netlist. Subsequently, we start an incremental placer based on such physical information to do both global and detailed placement on the clustered netlist. Finally, after unclustering we use the 
detailed placement algorithm to refine the layout.

Based on this simple two-phase placement flow, we implement a high-quality analytical placement algorithm, SCPlace (see Fig. 5). We use SafeChoice as the clustering algorithm inside SCPlace. As mentioned above, non-physical clustering may produce some low-quality clusters due to the lack of physical information, so we want SafeChoice to generate less such clusters. Therefore, we set $C_{t}=16$ instead of $C_{t}=21$. For the Physical SafeChoice in the second phase, we use the default $C_{t}=21$. mPL6 [5] is applied as the placement engine. However, mPL6 is based on a multilevel framework, and uses $\mathrm{BC}$ as its internal clustering algorithm. Without turning off BC inside mPL6, we cannot demonstrate the effectiveness of SafeChoice, because the internal clustering process will produce some noise to the results. Therefore, we turn off the BC clustering inside mPL6 by adding "-cluster_ratio 1" to the command line, so that mPL6 performs only one-level placement without any clustering inside, i.e., flat-mPL6 ${ }^{5}$. The detailed placer FastDP [6] is used as the additional detailed placement algorithm in SCPlace.

\section{EXPERIMENTAL RESULTS}

All experiments are run on a Linux server with Intel Xeon 2.83 GHz CPU and $32 \mathrm{~GB}$ memory. ISPD 05/06 placement benchmarks [21], [22] are used as the test circuits. For the ISPD 06 circuits, we use the density-penalty scaled HPWL defined in [22]. Firstly, we show the comparison of various clustering algorithms for different clustering objectives. Secondly, we compare SCPlace with the stat-of-the-art placement algorithms.

\section{A. Comparison of Clustering Algorithms}

We compare SC with three clustering algorithms FC [13], $\mathrm{BC}$ [16] and $\mathrm{NC}$ [17]. We implemented FC and BC by ourselves and obtained the binary of NC from the authors [17]. For BC the lazy-update [16] is used to speed up its runtime.

In the experiments, the clustering algorithm is applied as a pre-processing step before placement (see Fig. 6). We adopt mPL6 [5] as the placer. Due to the same reason mentioned in Section V, we use flat-mPL6 here. In Fig. 6 after unclustering, we arrange the objects inside each cluster in one row. The order among those objects are random. Subsequently the locations of all objects are sent to flat-mPL6 for detailed placement. Because of the random order of objects within each cluster, flat-mPL6 detailed placer alone may not be enough to generate a good result. So we apply the detailed placer FastDP [6] to further refine the layout after flat-mPL6 detailed placement.

We normalize the results of flat-mPL6 with various preprocessing clustering to the results of flat-mPL6 without any pre-processing clustering. For fair comparison, FastDP is applied to further refine the output layouts from the flat-mPL6 without pre-processing clustering. We conduct five sets of experiments.

\footnotetext{
${ }^{5}$ As far as we know, mPL6 is the only placer that can turn off the interna clustering without modifying the source code.
}

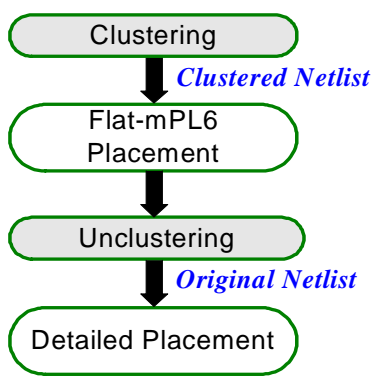

Fig. 6. Experimental flow for clustering algorithm.

I. Clustering Targeting at Safe Cluster: We compare SC$\mathrm{G}$ with FC and BC. FC's and BC's target $\gamma$ is set the same as SC-G's. Table IV shows that SC-G's HPWL is $2 \%$ worse than BC's and $1 \%$ better than FC's. For both clustering time and total time, SC-G is the fastest. Note that the cost $C$ of some unsafe (i.e., $S_{\max }>0$ ) clusters may be better than some safe clusters. But unfortunately SC-G does not form any unsafe clusters. This makes SC-G's HPWL worse than BC's.

II. Clustering Targeting at NetCluster's Clustering Ratio: In this set of experiments, we compare SC-R with FC, BC and NC based on NC's $\gamma$. Since NC terminates when no more clusters can be formed, it cannot reach any $\gamma$ as the users desire. For each circuit the target $\gamma$ of other algorithms is set the same as NC's. As shown in Table V, SC-R consistently generates the best HPWL for all 16 test cases, except for one case (bigblue3) where SC-R is $1 \%$ worse than BC. On average SC-R generates $4 \%, 1 \%$ and $5 \%$ better HPWL than FC, BC and $\mathrm{NC}$, respectively. In terms of clustering time, SC-R is $2.5 \times$ faster than BC, while $45 \%$ and $19 \%$ slower than FC and $\mathrm{NC}$, respectively. For the total time, SC-R is $1 \%$ and $7 \%$ faster than $\mathrm{FC}$ and $\mathrm{BC}$, while $5 \%$ slower than $\mathrm{NC}$.

III. Clustering Targeting at Various Clustering Ratios: We compare SC-R with FC and BC on five target clustering ratios $\gamma=0.2,0.3,0.4,0.5,0.6$. In Table VI the results are organized based on the circuits. We have two observations: (1) As $\gamma$ goes lower, the clustering time increases but the total time generally decreases; (2) To improve the HPWL, for some circuits (e.g., adaptec5) it is good to cluster more objects. But for some circuits (e.g., newblue2) low $\gamma$ degrades the HPWL. Fig. 7 shows the average normalized clustering time, HPWL and total time over all circuits for each $\gamma$. For clustering time, $\mathrm{SC}-\mathrm{R}$ is faster than $\mathrm{BC}$ for all $\gamma$, except for $\gamma=0.2$ where $\mathrm{SC}-\mathrm{R}$ is $12 \%$ slower. For all $\gamma$, SC-R consistently produces the best HPWL compared with both FC and $\mathrm{BC}$. Regarding the total time SC-R is consistently faster than BC. Even though $\mathrm{SC}-\mathrm{R}$ is slower than FC on clustering time, SC-R's total time is very comparable with FC's, which means clusters produced by SC-R are preferred by the placer. Furthermore, considering the significant HPWL improvements over FC and the small percentage of clustering time over total time, we believe such slow down is acceptable.

IV. Clustering Targeting at Best Placement Wirelength: Table VI shows that various $\gamma$ leads to various HPWL for each circuit. Here, we show that for most of the circuits, $\mathrm{SC}$ is able to automatically stop clustering, when the $\gamma$ for 
TABLE IV

COMPARISON With FIRstChOICE AND BESTCHOICE BASED ON SC-G's CLUSTERING RATIO (* COMPARISON OF SCALED HPWL).

\begin{tabular}{|c|c|c|c|c|c|c|c|c|c|c|c|c|}
\hline \multirow[t]{2}{*}{ Circuit } & \multicolumn{2}{|c|}{ Flat-mPL6 } & \multirow{2}{*}{$\begin{array}{l}\text { Clustering } \\
\text { Ratio }(\gamma)\end{array}$} & \multicolumn{3}{|c|}{ Clustering Time (s) } & \multicolumn{3}{|c|}{ Normalized HPWL to Flat-mPL6 } & \multicolumn{3}{|c|}{ Normalized Total Time to Flat-mPL6 } \\
\hline & HPWL $(\times 10 e 6)$ & Time $(\mathrm{s})$ & & FC & $\mathrm{BC}$ & SC-G & $\mathrm{FC}$ & $\mathrm{BC}$ & SC-G & $\mathrm{FC}$ & $\mathrm{BC}$ & SC-G \\
\hline adaptec1 & 78.91 & 1197 & 0.80 & 1 & 2 & 8 & 1.00 & 1.00 & 1.00 & 1.61 & 1.47 & 1.24 \\
\hline adaptec2 & 90.71 & 1241 & 0.77 & 2 & 4 & 10 & 0.99 & 0.99 & 1.00 & 1.73 & 1.63 & 1.43 \\
\hline adaptec 3 & 210.34 & 3923 & 0.71 & 6 & 23 & 33 & 1.00 & 0.99 & 0.99 & 1.38 & 1.51 & 1.17 \\
\hline adaptec4 & 188.39 & 3463 & 0.62 & 9 & 24 & 38 & 1.00 & 0.99 & 0.98 & 1.76 & 1.70 & 1.28 \\
\hline bigblue 1 & 96.73 & 1424 & 0.77 & 2 & 4 & 11 & 0.99 & 0.99 & 1.00 & 1.61 & 1.46 & 1.60 \\
\hline bigblue2 & 146.98 & 3988 & 0.73 & 142 & 605 & 101 & 1.00 & 0.99 & 0.99 & 1.57 & 1.54 & 1.52 \\
\hline bigblue 3 & 419.56 & 9486 & 0.58 & 35 & 123 & 91 & 0.91 & 0.88 & 0.90 & 1.01 & 1.00 & 1.04 \\
\hline bigblue4 & 812.89 & 10543 & 0.64 & 273 & 1529 & 287 & 1.00 & 0.99 & 0.99 & 1.47 & 1.41 & 1.34 \\
\hline adaptec5* & 731.47 & 7892 & 0.68 & 60 & 263 & 95 & 0.87 & 0.74 & 0.81 & 1.04 & 1.20 & 1.14 \\
\hline newblue $1 *$ & 109.85 & 17305 & 0.78 & 48 & 294 & 53 & 0.98 & 0.93 & 1.00 & 1.25 & 1.41 & 1.07 \\
\hline newblue $2 *$ & 197.44 & 4396 & 0.68 & 19 & 62 & 57 & 1.00 & 0.99 & 0.99 & 1.04 & 0.95 & 1.06 \\
\hline newblue $3 *$ & 320.63 & 10200 & 0.65 & 337 & 2393 & 228 & 0.94 & 0.96 & 0.95 & 1.29 & 1.65 & 1.67 \\
\hline newblue4* & 438.99 & 7779 & 0.71 & 30 & 137 & 48 & 0.92 & 0.88 & 0.95 & 0.89 & 0.85 & 0.90 \\
\hline newblue $5^{*}$ & 836.62 & 10124 & 0.66 & 363 & 1728 & 112 & 0.99 & 0.83 & 0.91 & 1.46 & 1.44 & 1.10 \\
\hline newblue6* & 520.95 & 7575 & 0.74 & 572 & 3487 & 204 & 0.99 & 0.98 & 0.98 & 1.78 & 2.14 & 1.42 \\
\hline newblue $7 *$ & 1076.36 & 19219 & 0.64 & 124 & 367 & 181 & 0.98 & 0.97 & 0.97 & 1.20 & 1.23 & 1.15 \\
\hline \multicolumn{4}{|c|}{ Average Normalized } & 1.006 & 5.303 & $\mathbf{1}$ & 0.974 & 0.944 & 0.963 & 1.381 & 1.413 & 1.258 \\
\hline
\end{tabular}

TABLE V

COMPARison With FirstChoice, BestChoice AND NetCluster BASEd On NetCluster's Clustering RATIO (* COMPARISON OF SCALED HPWL).

\begin{tabular}{|c|c|c|c|c|c|c|c|c|c|c|c|c|c|c|c|}
\hline \multirow[t]{2}{*}{ Circuit } & \multicolumn{2}{|c|}{ Flat-mPL6 } & \multirow{2}{*}{$\begin{array}{l}\text { Clustering } \\
\text { Ratio }(\gamma)\end{array}$} & \multicolumn{4}{|c|}{ Clustering Time (s) } & \multicolumn{4}{|c|}{ Normalized HPWL to Flat-mPL6 } & \multicolumn{4}{|c|}{ Normalized Total Time to Flat-mPL6 } \\
\hline & HPWL $(\times 10 e 6)$ & Time $(\mathrm{s})$ & & FC & $\mathrm{BC}$ & $\mathrm{NC}$ & SC-R & $\mathrm{FC}$ & $\mathrm{BC}$ & $\mathrm{NC}$ & SC-R & $\mathrm{FC}$ & $\mathrm{BC}$ & $\mathrm{NC}$ & SC-R \\
\hline adaptec1 & 78.91 & 1197 & 0.6381 & 1 & 3 & 69 & 20 & 1.00 & 1.00 & 1.01 & 0.99 & 0.92 & 0.91 & 1.15 & 1.04 \\
\hline adaptec 2 & 90.71 & 1241 & 0.5764 & 2 & 6 & 63 & 30 & 1.01 & 1.00 & 1.00 & 0.99 & 1.22 & 1.11 & 1.11 & 1.28 \\
\hline adaptec 3 & 210.34 & 3923 & 0.5677 & 7 & 24 & 62 & 98 & 1.02 & 0.99 & 0.99 & 0.99 & 1.15 & 1.09 & 1.00 & 1.04 \\
\hline adaptec4 & 188.39 & 3463 & 0.5382 & 8 & 26 & 58 & 86 & 1.01 & 1.00 & 0.98 & 0.98 & 1.19 & 1.13 & 1.07 & 1.15 \\
\hline bigblue1 & 96.73 & 1424 & 0.6128 & 2 & 5 & 66 & 23 & 0.99 & 0.98 & 0.98 & 0.98 & 1.19 & 1.08 & 1.21 & 1.13 \\
\hline bigblue2 & 146.98 & 3988 & 0.5977 & 195 & 814 & 64 & 181 & 1.02 & 1.00 & 0.99 & 0.99 & 0.98 & 1.11 & 0.89 & 0.86 \\
\hline bigblue 3 & 419.56 & 9486 & 0.5074 & 36 & 144 & 53 & 163 & 0.92 & 0.87 & 0.89 & 0.88 & 0.81 & 0.81 & 0.74 & 0.76 \\
\hline bigblue4 & 812.89 & 10543 & 0.5617 & 315 & 1696 & 58 & 588 & 1.01 & 0.99 & 0.99 & 0.99 & 1.21 & 1.27 & 1.10 & 1.19 \\
\hline adaptec5* & 731.47 & 7892 & 0.5569 & 81 & 335 & 60 & 284 & 0.87 & 0.73 & 0.79 & 0.69 & 0.98 & 0.98 & 0.90 & 0.92 \\
\hline newblue $1^{*}$ & 109.85 & 17305 & 0.5674 & 90 & 472 & 62 & 125 & 0.93 & 0.90 & 1.03 & 0.86 & 0.82 & 0.88 & 0.77 & 0.83 \\
\hline newblue2* & 197.44 & 4396 & 0.5886 & 22 & 65 & 65 & 92 & 1.02 & 1.00 & 1.10 & 1.00 & 0.74 & 0.81 & 0.69 & 0.77 \\
\hline newblue $3^{*}$ & 320.63 & 10200 & 0.5462 & 427 & 2440 & 63 & 342 & 0.93 & 0.93 & 1.15 & 0.93 & 1.04 & 1.39 & 0.99 & 1.04 \\
\hline newblue $4 *$ & 438.99 & 7779 & 0.6357 & 34 & 159 & 68 & 109 & 0.92 & 0.86 & 0.93 & 0.85 & 0.63 & 0.62 & 0.59 & 0.58 \\
\hline newblue $5^{*}$ & 836.62 & 10124 & 0.5505 & 481 & 1860 & 58 & 214 & 0.92 & 0.81 & 0.84 & 0.79 & 1.08 & 1.07 & 0.95 & 1.13 \\
\hline newblue6* & 520.95 & 7575 & 0.5836 & 868 & 4871 & 64 & 755 & 0.99 & 0.97 & 0.97 & 0.97 & 1.14 & 1.78 & 1.01 & 1.05 \\
\hline newblue $7 *$ & 1076.36 & 19219 & 0.5634 & 142 & 423 & 60 & 519 & 0.99 & 0.97 & 0.99 & 0.97 & 0.89 & 0.87 & 0.89 & 0.98 \\
\hline \multicolumn{4}{|c|}{ Average Normalized } & 0.545 & 2.475 & 0.813 & 1 & 0.971 & 0.937 & 0.978 & 0.928 & 1.000 & 1.056 & 0.940 & 0.985 \\
\hline
\end{tabular}

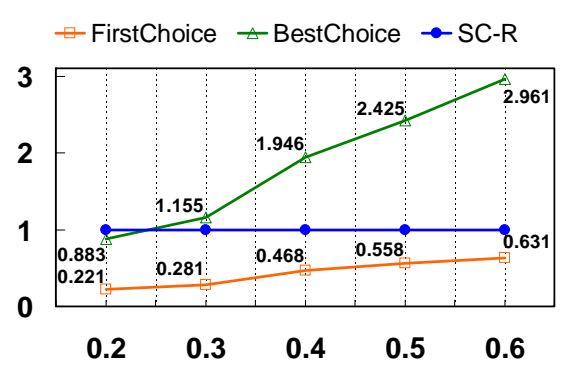

(a) Normalized clustering time to SC-R

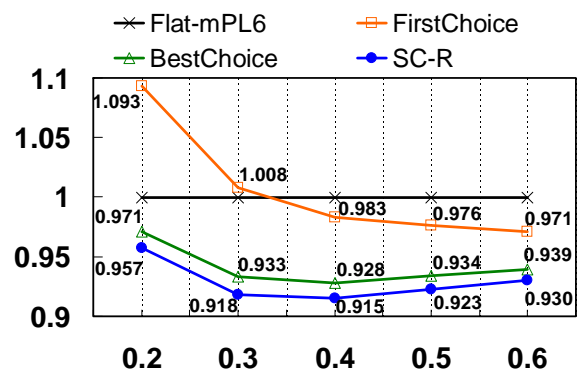

(b) Normalized HPWL to flat-mPL6

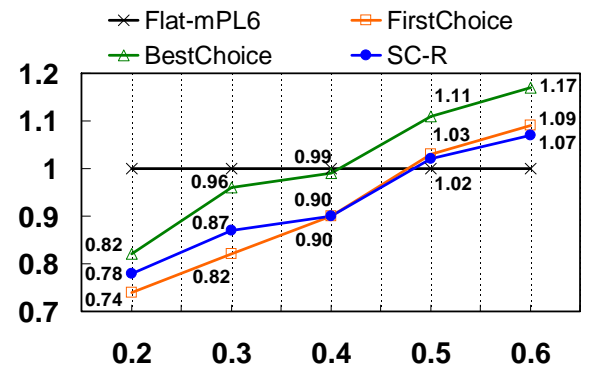

(c) Normalized total time to flat-mPL6

Fig. 7. Average normalized clustering time, HPWL and total time over all circuits for target $\gamma=0.2,0.3,0.4,0.5,0.6$.

the best HPWL is reached (see Table VII). Readers may compare Table VI and Table VII to verify this. To see how one-level clustering compares with multilevel clustering, we generate the results of original multilevel mPL6 with FastDP ("mPL6+FastDP") in Table VII. The clustering time and final $\gamma$ inside mPL6 are listed in Table VII. We can see that mPL6 has 4 levels of clustering and placement. Comparing SC with "mPL6+FastDP", even though SC on average generates $3 \%$ worse HWPL, for almost half of the circuits SC's HPWL is even better than "mPL6+FastDP". For most circuits, the
HPWL generated by SC and "mPL6+FastDP" are very comparable. Regarding the total time, $\mathrm{SC}$ is significantly faster than "mPL6+FastDP" by 33\%. Such results show that for some circuits one-level SC clustering generates better HPWL than multilevel BC clustering with substantial runtime speedup. From that we see prospective improvements if SC is applied into the multilevel placement framework. 
TABLE VI

COMPARISON WITH FIRSTCHOICE AND BESTCHOICE ON TARGET $\gamma=0.2,0.3,0.4,0.5,0.6$ (* COMPARISON OF SCALED HPWL).

\begin{tabular}{|c|c|c|c|c|c|c|c|c|c|c|c|c|}
\hline \multirow[t]{2}{*}{ Circuit } & \multicolumn{2}{|c|}{ Flat-mPL6 } & Clustering & Clu & ring $\mathrm{T}$ & e (s) & Norm & ized I & Flat-mPL6 & Nori & ized 7 & to Flat-mPL6 \\
\hline & HPWL $(\times 10 e 6)$ & Time (s) & Ratio $(\gamma)$ & $\mathrm{FC}$ & $\mathrm{BC}$ & SC-R & $\mathrm{FC}$ & $\mathrm{BC}$ & SC-R & $\mathrm{FC}$ & $\mathrm{BC}$ & SC-R \\
\hline & & & 0.2 & 4 & 8 & 187 & 1.12 & 1.06 & 1.03 & 0.94 & 0.80 & 0.88 \\
\hline & & & 0.3 & 3 & 6 & 121 & 1.05 & 1.02 & 1.00 & 0.90 & 0.80 & 0.92 \\
\hline adaptec 1 & 78.91 & 1197 & 0.4 & 2 & 5 & 51 & 1.01 & 1.00 & 0.99 & 0.93 & 0.86 & 1.00 \\
\hline & & & 0.5 & 2 & 4 & 35 & 1.00 & 1.00 & 0.99 & 0.95 & 0.92 & 0.91 \\
\hline & & & 0.6 & 2 & 3 & 24 & 1.00 & 0.99 & 0.99 & 1.04 & 1.02 & 1.04 \\
\hline & & & 0.2 & 8 & 16 & 238 & 1.08 & 1.02 & 1.00 & 1.03 & 0.82 & 1.01 \\
\hline & & & 0.3 & 5 & 12 & 144 & 1.05 & 0.99 & 0.98 & 1.35 & 1.20 & 1.22 \\
\hline adaptec2 & 90.71 & 1241 & 0.4 & 4 & 9 & 59 & 1.03 & 1.01 & 0.98 & 1.38 & 1.19 & 1.23 \\
\hline & & & 0.5 & 3 & 7 & 39 & 1.01 & 1.00 & 0.98 & 1.43 & 1.19 & 1.28 \\
\hline & & & 0.6 & 3 & 6 & 26 & 1.00 & 0.99 & 0.98 & 1.45 & 1.24 & 1.20 \\
\hline & & & 0.2 & 19 & 46 & 572 & 1.15 & 1.02 & 1.02 & 0.77 & 0.70 & 0.76 \\
\hline & & & 0.3 & 14 & 38 & 390 & 1.08 & 1.00 & 0.99 & 0.79 & 0.72 & 0.81 \\
\hline adaptec 3 & 210.34 & 3923 & 0.4 & 11 & 32 & 162 & 1.04 & 1.00 & 0.99 & 0.94 & 0.74 & 0.73 \\
\hline & & & 0.5 & 9 & 26 & 114 & 1.04 & 1.00 & 0.98 & 1.28 & 1.16 & 1.17 \\
\hline & & & 0.6 & 7 & 23 & 80 & 1.01 & 1.00 & 0.98 & 1.35 & 1.21 & 1.23 \\
\hline & & & 0.2 & 16 & 49 & 403 & 1.08 & 0.99 & 0.99 & 0.81 & 0.70 & 0.79 \\
\hline & & & 0.3 & 13 & 42 & 276 & 1.04 & 0.98 & 0.98 & 0.82 & 0.74 & 0.77 \\
\hline adaptec 4 & 188.39 & 3463 & 0.4 & 11 & 35 & 130 & 1.02 & 0.99 & 0.98 & 0.83 & 0.75 & 0.85 \\
\hline & & & 0.5 & 9 & 30 & 89 & 1.01 & 0.99 & 0.98 & 1.28 & 1.26 & 1.18 \\
\hline & & & 0.6 & 7 & 22 & 59 & 1.00 & 0.99 & 0.99 & 1.16 & 1.29 & 1.18 \\
\hline & & & 0.2 & 8 & 15 & 297 & 1.05 & 1.01 & 1.02 & 0.86 & 0.68 & 0.98 \\
\hline & & & 0.3 & 5 & 12 & 179 & 1.02 & 0.98 & 0.99 & 0.85 & 0.95 & 0.98 \\
\hline bigblue1 & 96.73 & 1424 & 0.4 & 4 & 9 & 66 & 1.01 & 0.98 & 0.98 & 0.91 & 0.86 & 0.91 \\
\hline & & & 0.5 & 3 & 7 & 39 & 1.00 & 0.97 & 0.98 & 1.01 & 0.85 & 0.89 \\
\hline & & & 0.6 & 2 & 6 & 23 & 1.00 & 0.98 & 0.98 & 1.09 & 1.17 & 1.18 \\
\hline & & & 0.2 & 395 & 1749 & 1162 & 1.15 & 1.07 & 1.05 & 0.84 & 1.05 & 0.92 \\
\hline & & & 0.3 & 344 & 1516 & 667 & 1.07 & 1.01 & 1.01 & 0.86 & 1.16 & 0.91 \\
\hline bigblue2 & 146.98 & 3988 & 0.4 & 302 & 1295 & 359 & 1.04 & 1.00 & 0.99 & 0.88 & 1.14 & 0.90 \\
\hline & & & 0.5 & 244 & 1005 & 226 & 1.03 & 0.99 & 0.99 & 0.95 & 1.18 & 0.92 \\
\hline & & & 0.6 & 194 & 796 & 159 & 1.01 & 1.00 & 0.99 & 1.09 & 1.11 & 0.99 \\
\hline & & & 0.2 & 69 & 264 & 800 & 0.92 & 0.82 & 0.85 & 0.52 & 0.49 & 0.57 \\
\hline & & & 0.3 & 55 & 221 & 492 & 0.92 & 0.87 & 0.83 & 0.78 & 0.76 & 0.75 \\
\hline bigblue 3 & 419.56 & 9486 & 0.4 & 46 & 174 & 241 & 0.92 & 0.84 & 0.85 & 0.90 & 0.81 & 0.82 \\
\hline & & & 0.5 & 36 & 146 & 158 & 0.93 & 0.88 & 0.88 & 0.93 & 0.95 & 0.91 \\
\hline & & & 0.6 & 30 & 116 & 89 & 0.91 & 0.88 & 0.89 & 1.00 & 1.01 & 0.91 \\
\hline & & & 0.2 & 633 & 2907 & 3262 & 1.12 & 1.01 & 1.02 & 1.06 & 1.17 & 1.19 \\
\hline & & & 0.3 & 534 & 2576 & 2220 & 1.06 & 1.00 & 0.99 & 1.19 & 1.44 & 1.24 \\
\hline bigblue4 & 812.89 & 10543 & 0.4 & 451 & 2169 & 1145 & 1.03 & 0.99 & 0.99 & 1.16 & 1.45 & 1.14 \\
\hline & & & 0.5 & 368 & 1819 & 733 & 1.01 & 0.99 & 0.99 & 1.24 & 1.35 & 1.23 \\
\hline & & & 0.6 & 288 & 1453 & 434 & 1.01 & 0.99 & 0.99 & 1.27 & 1.38 & 1.22 \\
\hline & & & 0.2 & 165 & 569 & 1424 & 0.77 & 0.63 & 0.62 & 0.52 & 0.56 & 0.66 \\
\hline & & & 0.3 & 139 & 503 & 984 & 0.83 & 0.66 & 0.63 & 0.52 & 0.52 & 0.62 \\
\hline adaptec $5^{*}$ & 731.47 & 7892 & 0.4 & 114 & 419 & 456 & 0.84 & 0.68 & 0.65 & 0.61 & 0.58 & 0.59 \\
\hline & & & 0.5 & 93 & 358 & 324 & 0.86 & 0.72 & 0.69 & 1.07 & 1.29 & 1.07 \\
\hline & & & 0.6 & 73 & 311 & 204 & 0.88 & 0.73 & 0.70 & 1.23 & 1.32 & 1.15 \\
\hline & & & 0.2 & 169 & 806 & 781 & 0.91 & 0.88 & 0.81 & 0.13 & 0.24 & 0.23 \\
\hline & & & 0.3 & 149 & 718 & 527 & 0.91 & 0.86 & 0.80 & 0.23 & 0.49 & 0.41 \\
\hline newblue $1 *$ & 109.85 & 17305 & 0.4 & 127 & 630 & 226 & 0.91 & 0.87 & 0.81 & 0.30 & 0.57 & 0.39 \\
\hline & & & 0.5 & 104 & 538 & 141 & 0.93 & 0.89 & 0.84 & 0.91 & 1.30 & 0.96 \\
\hline & & & 0.6 & 84 & 434 & 93 & 0.94 & 0.90 & 0.86 & 1.05 & 1.31 & 1.04 \\
\hline & & & 0.2 & 43 & 200 & 415 & 2.16 & 1.58 & 1.44 & 0.74 & 0.69 & 0.81 \\
\hline & & & 0.3 & 37 & 181 & 278 & 1.29 & 1.11 & 1.11 & 0.86 & 0.88 & 0.76 \\
\hline newblue $2 *$ & 197.44 & 4396 & 0.4 & 32 & 164 & 155 & 1.07 & 1.03 & 1.03 & 0.82 & 0.89 & 0.86 \\
\hline & & & 0.5 & 26 & 128 & 116 & 1.02 & 1.01 & 1.01 & 1.04 & 0.88 & 0.97 \\
\hline & & & 0.6 & 21 & 65 & 84 & 1.01 & 1.00 & 1.00 & 0.90 & 0.89 & 0.90 \\
\hline & & & 0.2 & 931 & 3789 & 1010 & 0.98 & 0.89 & 0.89 & 0.50 & 0.76 & 0.50 \\
\hline & & & 0.3 & 783 & 3480 & 692 & 0.93 & 0.89 & 0.90 & 0.90 & 1.60 & 1.00 \\
\hline newblue $3 *$ & 320.63 & 10200 & 0.4 & 630 & 3041 & 407 & 0.92 & 0.90 & 0.91 & 0.95 & 1.56 & 1.14 \\
\hline & & & 0.5 & 487 & 2546 & 326 & 0.92 & 0.93 & 0.93 & 1.00 & 1.34 & 1.15 \\
\hline & & & 0.6 & 362 & 2299 & 256 & 0.94 & 0.95 & 0.95 & 1.05 & 1.41 & 1.23 \\
\hline & & & 0.2 & 77 & 334 & 981 & 0.94 & 0.88 & 0.81 & 0.46 & 0.57 & 0.49 \\
\hline & & & 0.3 & 66 & 302 & 643 & 0.91 & 0.88 & 0.80 & 0.50 & 0.64 & 0.60 \\
\hline newblue 4* & 438.99 & 7779 & 0.4 & 55 & 267 & 275 & 0.91 & 0.86 & 0.81 & 0.59 & 0.72 & 0.62 \\
\hline & & & 0.5 & 46 & 221 & 188 & 0.92 & 0.86 & 0.81 & 0.53 & 0.61 & 0.57 \\
\hline & & & 0.6 & 37 & 168 & 114 & 0.93 & 0.85 & 0.83 & 0.62 & 0.59 & 0.63 \\
\hline & & & 0.2 & 1093 & 3948 & 1483 & 0.94 & 0.70 & 0.78 & 0.64 & 1.02 & 0.62 \\
\hline & & & 0.3 & 877 & 2863 & 935 & 0.95 & 0.73 & 0.74 & 0.71 & 0.95 & 0.69 \\
\hline newblue 5* & 836.62 & 10124 & 0.4 & 693 & 2124 & 392 & 0.96 & 0.77 & 0.77 & 1.09 & 1.10 & 1.01 \\
\hline & & & 0.5 & 532 & 1903 & 237 & 0.94 & 0.78 & 0.77 & 1.04 & 1.10 & 0.98 \\
\hline & & & 0.6 & 399 & 1713 & 155 & 0.92 & 0.82 & 0.80 & 1.04 & 1.10 & 0.94 \\
\hline & & & 0.2 & 1941 & 8229 & 4058 & 1.05 & 0.99 & 0.97 & 1.16 & 1.95 & 1.19 \\
\hline & & & 0.3 & 1641 & 7415 & 2793 & 1.01 & 0.97 & 0.96 & 1.11 & 1.77 & 1.31 \\
\hline newblue6* & 520.95 & 7575 & 0.4 & 1343 & 6558 & 1378 & 1.00 & 0.97 & 0.96 & 1.27 & 1.88 & 1.28 \\
\hline & & & 0.5 & 1082 & 5391 & 890 & 0.99 & 0.97 & 0.97 & 1.14 & 1.64 & 1.07 \\
\hline & & & 0.6 & 824 & 4535 & 639 & 0.99 & 0.98 & 0.97 & 1.17 & 1.66 & 1.16 \\
\hline & & & 0.2 & 290 & 948 & 2704 & 1.07 & 0.99 & 1.00 & 0.90 & 0.98 & 0.81 \\
\hline & & & 0.3 & 238 & 738 & 1774 & 1.02 & 0.97 & 0.97 & 0.78 & 0.80 & 0.93 \\
\hline newblue $7 *$ & 1076.36 & 19219 & 0.4 & 197 & 605 & 891 & 1.00 & 0.97 & 0.97 & 0.79 & 0.74 & 0.95 \\
\hline & & & 0.5 & 159 & 472 & 596 & 0.99 & 0.97 & 0.97 & 0.76 & 0.72 & 1.00 \\
\hline & & & 0.6 & 126 & 380 & 422 & 0.98 & 0.97 & 0.97 & 0.93 & 1.00 & 1.03 \\
\hline
\end{tabular}


TABLE VII

COMPARISON WITH MULTILEVEL MPL6 (* COMPARISON OF SCALED HPWL).

\begin{tabular}{|c|c|c|c|c|c|c|c|c|c|c|c|}
\hline \multirow[t]{2}{*}{ Circuit } & \multicolumn{3}{|c|}{ HPWL $(\times 10 e 6)$} & \multicolumn{3}{|c|}{ Total Time (s) } & \multicolumn{2}{|c|}{ SC Clustering Info. } & \multicolumn{3}{|c|}{ BC Clustering Info. inside mPL6 } \\
\hline & Flat-mPL6 & $\mathrm{SC}$ & mPL6+FastDP & Flat-mPL6 & SC & mPL6+FastDP & Time (s) & $\gamma$ & Time (s) & Final $\gamma$ & \# of levels \\
\hline adaptec 1 & 78.91 & 78.51 & $\mathbf{7 6 . 4 7}$ & 1197 & 1238 & 1807 & 76 & 0.33 & 29 & 0.006 & 4 \\
\hline adaptec 2 & 90.71 & 88.51 & 89.19 & 1241 & 2064 & 2032 & 73 & 0.36 & 47 & 0.006 & 4 \\
\hline adaptec 3 & 210.34 & 207.27 & 206.00 & 3923 & 3732 & 6187 & 228 & 0.33 & 87 & 0.006 & 4 \\
\hline adaptec 4 & 188.39 & 184.33 & 187.51 & 3463 & 3227 & 5687 & 208 & 0.31 & 67 & 0.007 & 4 \\
\hline bigblue1 & 96.73 & 95.31 & 95.14 & 1424 & 1319 & 2208 & 109 & 0.32 & 42 & 0.008 & 4 \\
\hline bigblue2 & 146.98 & 146.07 & 146.57 & 3988 & 4183 & 5992 & 458 & 0.36 & 81 & 0.045 & 4 \\
\hline bigblue 3 & 419.56 & 357.56 & 331.70 & 9486 & 10516 & 8842 & 420 & 0.30 & 131 & 0.005 & 4 \\
\hline bigblue4 & 812.89 & 803.43 & 806.83 & 10543 & 15460 & 19457 & 1622 & 0.33 & 468 & 0.008 & 4 \\
\hline newblue ${ }^{*}$ & 109.85 & 88.10 & 64.72 & 17305 & 7490 & 2567 & 368 & 0.31 & 44 & 0.005 & 4 \\
\hline newblue $2 *$ & 197.44 & 198.35 & 198.90 & 4396 & 6303 & 7141 & 91 & 0.58 & 61 & 0.007 & 4 \\
\hline newblue $3 *$ & 320.63 & 287.76 & 283.25 & 10200 & 14986 & 9644 & 683 & 0.30 & 66 & 0.029 & 4 \\
\hline newblue $4 *$ & 438.99 & 351.02 & 301.89 & 7779 & 6053 & 9481 & 421 & 0.33 & 93 & 0.010 & 4 \\
\hline newblue ${ }^{*}$ & 836.62 & 624.26 & 526.98 & 10124 & 8405 & 16220 & 625 & 0.34 & 251 & 0.008 & 4 \\
\hline newblue6* & 520.95 & 498.44 & 516.43 & 7575 & 11081 & 13566 & 2059 & 0.33 & 255 & 0.009 & 4 \\
\hline newblue $7 *$ & 1076.36 & 1042.97 & 1070.08 & 19219 & 21049 & 32561 & 1159 & 0.34 & 278 & 0.014 & 4 \\
\hline Normalized & $\overline{1}$ & 0.910 & 0.879 & 1 & 1.086 & 1.412 & & & & & \\
\hline
\end{tabular}

\section{B. Comparison of Placement Algorithms}

In this subsection, we compare SCPlace with the state-ofthe-art placement algorithms.

I. Firstly, we compare SCPlace with mPL6. We run both algorithms on the same machine. The results and the clustering information inside SCPlace are shown in Table VIII. In terms of the HPWL, SCPlace is consistently better than mPL6, except for one circuit (i.e., newblue1). On average, SCPlace generates $4 \%$ better HPWL than mPL6. Regarding the total runtime, SCPlace is $55 \%$ faster than mPL6. As mentioned in Section V, in the first phase of SCPlace we set $C_{t}=16$ rather than $C_{t}=21$ for non-physical SafeChoice, so that the nonphysical SafeChoice will stop clustering earlier to generate less low-quality clusters.

II. Secondly, we compare SCPlace with all other placement algorithms. Because some of the placers' binaries are not publicly available, instead of running every placer on the same machine, we directly cite the results from [7]. As far as we know, RQL [7] is the latest published placement algorithm in academic area, and it generates the best results on average compared with all previous placers. The experimental results are shown in Table IX. The "Previously Best" column shows the previously best HPWL achieved by other placers for each circuit. The results are quite promising. Regarding the HPWL, SCPlace is $2 \%, 25 \%, 9 \%, 6 \%, 3 \%, 21 \%, 6 \%$ and $1 \%$ better than NTUplace3, Capo10.5, mFAR, APlace, mPL6, Dragon, Kraftwerk and RQL, respectively. Even though SCPlace is $1 \%$ worse than the previously best approach, for 11 out of 16 circuits, SCPlace generates better results, which means SCPlace has broken the records for 11 circuits. All of the placers here, except for Kraftwerk, have at least four levels of placement. Using only one level of clustering and two phases of placement, SCPlace is able to beat all of them.

The runtime breakdown of SCPlace is presented in Table X. It shows that the total runtime is dominated by two steps, i.e., global placement and incremental placement. Both nonphysical and physical clustering contribute only $6 \%$ of the total runtime.
TABLE X

THE RUNTIME BREAKDOWN OF SCPLACE.

\begin{tabular}{|c|c|}
\hline Steps in SCPlace & Runtime $\%$ \\
\hline \hline Non-Physical Clustering & $6 \%$ \\
\hline Global Placement & $39 \%$ \\
\hline Physical Clustering & $6 \%$ \\
\hline Incremental Placement & $45 \%$ \\
\hline Detailed Placement & $4 \%$ \\
\hline
\end{tabular}

\section{CONCLUSION}

In this paper, we have presented SafeChoice, a novel highquality clustering algorithm. We aim at solving the fundamental problem - How to form safe clusters for placement. The clusters produced by SafeChoice are definitely essential for the placer to produce a good placement. Comprehensive experimental results show that SafeChoice is capable of producing the best clusters for the placer. Based on SafeChoice, we derived Physical SafeChoice, and integrated it into a highquality analytical placer, SCPlace. Promisingly, by a simple two-phase of placement, SCPlace significantly outperforms all state-of-the-art placement algorithms.

Our future work includes three directions: 1) To derive the safe condition for more than two vertices; 2) To develop our own placer based on SafeChoice, rather than feeding the clustered netlist to flat-mPL6 binary; 3) To integrate SafeChoice into other algorithms, e.g., hypergraph partitioning. Regarding the last point, we can simply integrate SafeChoice into existing partitioner. Or more interestingly, we can propose a safe condition for hypergraph partitioning, e.g., what is the safe condition to do a partition?

Finally, the source code of SafeChoice is publicly available at [25].

\section{ACKNOWLEDGMENT}

The authors would like to thank Guojie Luo from University of California, Los Angeles Computer-Aided Design group and Logan Rakai from University of Calgary for the help with mPL6 and NetCluster, respectively. They are also grateful to the anonymous reviewers for their helpful suggestions and comments on this paper. 
TABLE VIII

COMPARISON WITH ORIGINAL MULTILEVEL MPL6 (* COMPARISON OF SCALED HPWL).

\begin{tabular}{|c|c|c|c|c|c|c|c|c|c|}
\hline \multirow{3}{*}{ Circuit } & \multicolumn{2}{|c|}{ HPWL $(\times 10 e 6)$} & \multicolumn{2}{|c|}{ Total Time (s) } & \multicolumn{5}{|c|}{ Clustering Info. inside SCPlace } \\
\hline & \multirow[b]{2}{*}{ mPL6 } & \multirow[b]{2}{*}{ SCPlace } & \multirow[b]{2}{*}{ mPL6 } & \multirow[b]{2}{*}{ SCPlace } & \multicolumn{2}{|c|}{ SafeChoice } & \multicolumn{2}{|c|}{ Physical SafeChoice } & \multirow[b]{2}{*}{ \# of levels } \\
\hline & & & & & Time $(\mathrm{s})$ & $\gamma$ & Time (s) & $\gamma$ & \\
\hline adaptec1 & 78.05 & 76.50 & 1769 & 937 & 54 & 0.43 & 109 & 0.34 & 1 \\
\hline adaptec2 & 91.76 & 86.30 & 1940 & 1504 & 52 & 0.44 & 101 & 0.36 & 1 \\
\hline adaptec3 & 214.29 & 204.10 & 5949 & 2981 & 167 & 0.42 & 192 & 0.34 & 1 \\
\hline adaptec 4 & 194.25 & 183.20 & 5487 & 2652 & 150 & 0.40 & 213 & 0.32 & 1 \\
\hline bigblue1 & 96.75 & 93.58 & 2158 & 1182 & 60 & 0.43 & 136 & 0.33 & 1 \\
\hline bigblue 2 & 152.33 & 144.39 & 5842 & 3345 & 333 & 0.43 & 313 & 0.36 & 1 \\
\hline bigblue 3 & 343.89 & 336.01 & 8382 & 7682 & 288 & 0.39 & 302 & 0.31 & 1 \\
\hline bigblue4 & 829.42 & 790.76 & 18590 & 12486 & 1219 & 0.42 & 1233 & 0.33 & 1 \\
\hline adaptec5* & 430.42 & 419.72 & 10714 & 5528 & 459 & 0.41 & 263 & 0.32 & 1 \\
\hline newblue $1 *$ & 73.21 & 77.27 & 2489 & 10798 & 218 & 0.41 & 55 & 0.36 & 1 \\
\hline newblue 2* & 201.63 & 194.66 & 7109 & 4642 & 54 & 0.70 & 79 & 0.61 & 1 \\
\hline newblue $3 *$ & 284.04 & 281.59 & 9508 & 13736 & 577 & 0.39 & 337 & 0.33 & 1 \\
\hline newblue4* & 302.04 & 295.98 & 9410 & 4272 & 288 & 0.43 & 64 & 0.38 & 1 \\
\hline newblue 5* & 536.29 & 522.71 & 16085 & 10149 & 407 & 0.43 & 201 & 0.37 & 1 \\
\hline newblue6* & 521.28 & 494.10 & 13457 & 10877 & 1481 & 0.42 & 1113 & 0.34 & 1 \\
\hline newblue7* & 1083.66 & 1035.15 & 32372 & 23356 & 1003 & 0.43 & 932 & 0.34 & 1 \\
\hline Normalized & 1.036 & 1 & 1.549 & 1 & & & & & \\
\hline
\end{tabular}

TABLE IX

HPWL COMPARISON WITH THE STATE-OF-THE-ART PLACEMENT ALGORITHMS (* COMPARISON OF SCALED HPWL, $\dagger$ RESULTS ARE TUNED FOR EACH CIRCUIT, THE RESULTS OF ALL OTHER PLACERS (EXCEPT KRAFTWERK2) ARE CITED FROM [7] AND — DENOTES UNAVAILABLE RESULTS IN [7]).

\begin{tabular}{|c|c|c|c|c|c|c|c|c|c|c|}
\hline Circuit & NTUplace3 [1] & Capo10.5 [2] & mFAR [3] & APlace [4] & mPL6 [5] & Dragon [23] & Kraftwerk2 [24] & RQL [7] & Previously Best & SCPlace \\
\hline adaptec1 & 80.93 & 91.28 & - & 78.35 & 77.91 & - & 82.43 & 77.82 & 77.82 & 76.50 \\
\hline adaptec 2 & 89.95 & 100.75 & $91.53 \dagger$ & $87.31 \dagger$ & 91.96 & $94.72 \dagger$ & 92.85 & 88.51 & 87.31 & 86.30 \\
\hline adaptec 3 & 214.20 & 228.47 & - & 218.52 & 214.05 & - & 227.22 & 210.96 & 210.96 & 204.10 \\
\hline adaptec4 & 193.74 & 208.35 & $190.84 \dagger$ & $187.65 \dagger$ & 194.23 & $200.88 \dagger$ & 199.43 & 188.86 & 187.65 & 183.20 \\
\hline bigblue1 & 97.28 & 108.60 & $97.70 \dagger$ & $94.64 \dagger$ & 96.79 & $102.39 \dagger$ & 97.67 & 94.98 & 94.64 & 93.58 \\
\hline bigblue2 & 152.20 & 162.92 & $168.70 \dagger$ & $143.82 \dagger$ & 152.33 & $159.71 \dagger$ & 154.74 & 150.03 & 143.82 & 144.39 \\
\hline bigblue 3 & 348.48 & 398.49 & $379.95 \dagger$ & $357.89 \dagger$ & 344.37 & $380.45 \dagger$ & 343.32 & 323.09 & 323.09 & 336.01 \\
\hline bigblue 4 & 829.16 & 965.30 & $876.28 \dagger$ & $833.21 \dagger$ & 829.35 & $903.96 \dagger$ & 852.40 & 797.66 & 797.66 & 790.76 \\
\hline adaptec $5 *$ & 448.58 & 494.64 & 476.28 & 520.97 & 431.14 & 500.74 & 449.48 & 443.28 & 431.14 & 419.72 \\
\hline newblue $1 *$ & 61.08 & 98.48 & 77.54 & 73.31 & 67.02 & 80.77 & 66.19 & 64.43 & 61.08 & 77.27 \\
\hline newblue $2 *$ & 203.39 & 309.53 & 212.90 & 198.24 & 200.93 & 260.83 & 206.53 & 199.60 & 198.24 & 194.66 \\
\hline newblue $3 *$ & 278.89 & 361.25 & 303.91 & 273.64 & 287.05 & 524.58 & 279.57 & 269.33 & 269.33 & 281.59 \\
\hline newblue 4* & 301.19 & 362.40 & 324.40 & 384.12 & 299.66 & 341.16 & 309.44 & 308.75 & 299.66 & 295.98 \\
\hline newblue ${ }^{*}$ & 509.54 & 659.57 & 601.27 & 613.86 & 540.67 & 614.23 & 563.15 & 537.49 & 509.54 & 522.71 \\
\hline newblue6* & 521.65 & 668.66 & 535.96 & 522.73 & 518.70 & 572.53 & 537.59 & 515.69 & 515.69 & 494.10 \\
\hline newblue $7 *$ & 1099.66 & 1518.75 & 1153.76 & 1098.88 & 1082.92 & 1410.54 & 1162.12 & 1057.79 & 1057.79 & 1035.15 \\
\hline Normalized & 1.02 & 1.25 & 1.09 & 1.06 & 1.03 & 1.21 & 1.06 & 1.01 & 0.99 & 1 \\
\hline
\end{tabular}

\section{REFERENCES}

[1] T.-C. Chen, Z.-W. Jiang, T.-C. Hsu, H.-C. Chen, and Y.-W. Chang. A high-quality mixed-size analytical placer considering preplaced blocks and density constraints. In Proc. ICCAD, pages 187-192, 2006.

[2] J. A. Roy, S. N. Adya, D. A. Papa, and I. L. Markov. Min-cut floorplacement. IEEE Trans. on Computer-Aided Design, 25(7):13131326, July 2006.

[3] B. Hu and M. Marek-Sadowska. Multilevel fixed-point-addition-based VLSI placement. IEEE Trans. on Computer-Aided Design, 24(8):11881203, August 2005.

[4] A. B. Kahng and Q. Wang. A faster implementation of APlace. In Proc. ISPD, pages 218-220, 2006.

[5] T. Chan, J. Cong, J. Shinnerl, K. Sze, and M. Xie. mPL6: Enhanced multilevel mixed-sized placement. In Proc. ISPD, pages 212-214, 2006.

[6] N. Viswanathan, M. Pan, and C. Chu. FastPlace 3.0: A fast multilevel quadratic placement algorithm with placement congestion control. In Proc. ASP-DAC, pages 135-140, 2007.

[7] N. Viswanathan, G.-J. Nam, C. Alpert, P. Villarrubia, H. Ren, and C. Chu. RQL: Global placement via relaxed quadratic spreading and linearization. In Proc. DAC, pages 453-458, 2007.

[8] J. Z. Yan, N. Viswanathan, and C. Chu. Handling complexities in modern large-scale mixed-size placement. In Proc. DAC, pages 436-441, 2009.

[9] C. J. Alpert and A. B. Kahng. Recent developments in netlist partitioning: A survey. Integration, the VLSI Journal, 19(1-2):1-81, August 1995.

[10] G. Karypis, R. Aggarwal, V. Kumar, and S. Shekhar. Multilevel hypergraph partitioning: application in VLSI domain. In Proc. DAC, pages 526-529, 1997.

[11] G. Karypis and V. Kumar. Multilevel k-way hypergraph partitioning. In Proc. DAC, pages 343-348, 1999.
[12] C. J. Alpert, J.-H. Huang, and A. B. Kahng. Multilevel k-way hypergraph partitioning. In Proc. DAC, pages 530-533, 1997.

[13] T. Chan, J. Cong, and K. Sze. Multilevel generalized force-directed method for circuit placement. In Proc. ISPD, pages 185-192, 2005.

[14] J. Cong and S. K. Lim. Edge separability-based circuit clustering with application to multilevel circuit partitioning. IEEE Trans. on ComputerAided Design, 23(3):346-357, March 2004.

[15] B. Hu and M. Marek-Sadowska. Fine granularity clustering-based placement. IEEE Trans. on Computer-Aided Design, 23(4):527-536, April 2004.

[16] G.-J. Nam, S. Reda, C. J. Alpert, P. G. Villarrubia, and A. B. Kahng. A fast hierarchical quadratic placement algorithm. IEEE Trans. on Computer-Aided Design, 25(4):678-691, April 2006.

[17] J. Li, L. Behjat, and J. Huang. An effective clustering algorithm for mixed-size placement. In Proc. ISPD, pages 111-118, 2007.

[18] C. M. Fiduccia and R. M. Mattheyses. A linear-time heuristic for improving network partitions. In Proc. DAC, pages 175-181, 1982.

[19] J. Z. Yan, C. Chu, and W.-K. Mak. SafeChoice: A novel clustering algorithm for wirelength-driven placement. In Proc. ISPD, pages 185192, 2010.

[20] H. Chen, C.-K. Cheng, N.-C. Chou, A. B. Kahng, J. F. MacDonald, P. Suaris, B. Yao, and Z. Zhu. An algebraic multigrid solver for analytical placement with layout based clustering. In Proc. DAC, pages 794-799, 2003.

[21] G.-J. Nam, C. J. Alpert, P. Villarrubia, B. Winter, and M. Yildiz. The ISPD2005 placement contest and benchmarks suite. In Proc. ISPD, pages 216-220, 2005.

[22] G.-J. Nam. ISPD 2006 placement contest: Benchmark suite and results. In Proc. ISPD, pages 167-167, 2006.

[23] T. Taghavi, X. Yang, B.-K. Choi, M. Wang, and M. Sarrafzadeh. 
Dragon2005: large-scale mixed-size placement tool. In Proc. ISPD, pages 245-247, 2005.

[24] P. Spindler, U. Schlichtmann, and F. M. Johannes. Kraftwerk2 - A fast force-directed quadratic placement approach using an accurate net model. IEEE Trans. on Computer-Aided Design, 27(8):1398-1411, August 2008.

[25] SafeChoice source code. http://www.public.iastate.edu/ zijunyan/.

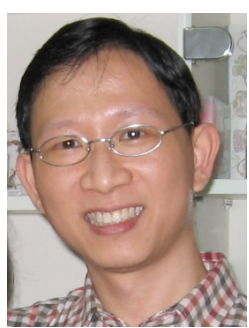

Wai-Kei Mak received the B.S. degree in computer science from the University of Hong Kong, Hong Kong, in 1993 and the M.S. and Ph.D. degrees in computer science from the University of Texas at Austin, U.S., in 1995 and 1998, respectively.

From 1999 to 2003, he was with the University of South Florida, U.S., as an Assistant Professor in the Department of Computer Science and Engineering. Since 2003, he has been with the Department of Computer Science of National Tsing Hua University, Taiwan, R.O.C., where he is currently an Associate Professor. His research interests include VLSI physical design automation, and FPGA architecture and CAD.

Dr. Mak has served on the program and/or the organizing committees of Asia South Pacific Design Automation Conference (ASP-DAC), International Conference on Field Programmable Logic and Applications (FPL), and International Conference on Field-Programmable Technology (FPT). He was the general chair of the 2008 International Conference on Field-Programmable Technology and was the technical program co-chair of the same conference in 2006. He is in the steering committee of the International Conference on Field-Programmable Technology since 2009.

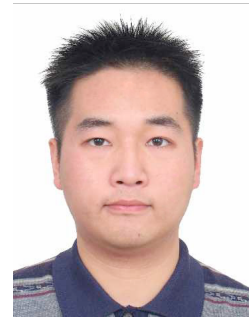

Jackey Z. Yan received the B.S. degree in automation from the Huazhong University of Science and Technology, Wuhan, China, in 2006. He is currently pursing the Ph.D. degree in computer engineering at the Department of Electrical and Computer Engineering, Iowa State University, Ames, IA.

Since November 2010, he has been working as Sr. Member of Technical Staff in the placement technology group at Cadence Design Systems Inc, San Jose, CA. His research interests include very large scale integration physical designs, specifically in algorithms for floorplanning and placement, and physical synthesis integrated system-on-a-chip designs.

Mr. Yan's work on fixed-outline floorplanning was nominated for the Best Paper Award at Design Automation Conference (DAC) 2008. His another work on hypergraph clustering for wirelength-driven placement was nominated for the Best Paper Award at International Symposium on Physical Design (ISPD) 2010. He received the Ultra-Excellent Student Award from Renesas Technology Corp., Tokyo, Japan, in 2005.

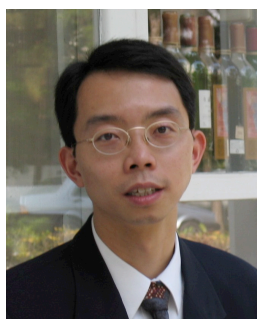

Chris Chu received the B.S. degree in computer science from the University of Hong Kong, Hong Kong, in 1993. He received the M.S. degree and the Ph.D. degree in computer science from the University of Texas at Austin in 1994 and 1999, respectively.

Dr. Chu is currently an Associate Professor in the Electrical and Computer Engineering Department at Iowa State University. His area of expertises include CAD of VLSI physical design, and design and analysis of algorithms. His recent research interests are performance-driven interconnect optimization and fast circuit floorplanning, placement, and routing algorithms.

He received the IEEE TCAD best paper award at 1999 for his work in performance-driven interconnect optimization. He received another IEEE TCAD best paper award at 2010 for his work in routing tree construction. $\mathrm{He}$ received the ISPD best paper award at 2004 for his work in efficient placement algorithm. He received the Bert Kay Best Dissertation Award for 1998-1999 from the Department of Computer Sciences in the University of Texas at Austin. 